\title{
POSITIVITY OF THE DENSITY FOR THE STOCHASTIC WAVE EQUATION IN TWO SPATIAL DIMENSIONS *,**
}

\author{
Mireille Chaleyat-Maurel ${ }^{1}$ And Marta Sanz-Solé ${ }^{1,2}$
}

\begin{abstract}
We consider the random vector $u(t, \underline{x})=\left(u\left(t, x_{1}\right), \ldots, u\left(t, x_{d}\right)\right)$, where $t>0, x_{1}, \ldots, x_{d}$ are distinct points of $\mathbb{R}^{2}$ and $u$ denotes the stochastic process solution to a stochastic wave equation driven by a noise white in time and correlated in space. In a recent paper by Millet and Sanz-Solé [10], sufficient conditions are given ensuring existence and smoothness of density for $u(t, \underline{x})$. We study here the positivity of such density. Using techniques developped in [1] (see also [9]) based on Analysis on an abstract Wiener space, we characterize the set of points $y \in \mathbb{R}^{d}$ where the density is positive and we prove that, under suitable assumptions, this set is $\mathbb{R}^{d}$.
\end{abstract}

Mathematics Subject Classification. 60H15, 60H07.

Received January 8, 2002.

\section{INTRODUCTION}

Consider the stochastic wave equation with two-dimensional spatial variable

$$
\begin{aligned}
\left(\frac{\partial^{2}}{\partial t^{2}}-\Delta\right) u(t, x) & =\sigma(u(t, x)) F(\mathrm{~d} t, \mathrm{~d} x)+b(u(t, x)), \\
u(0, x) & =u_{0}(x), \\
\frac{\partial u}{\partial t}(0, x) & =v_{0}(x)
\end{aligned}
$$

$(t, x) \in\left[0, \infty\left[\times \mathbb{R}^{2}\right.\right.$.

The coefficients $\sigma$ and $b$ are $\mathcal{C}^{\infty}$ functions with bounded derivatives of any order $i \geq 1$. The noise $F(t, x)$ is supposed to be a martingale measure (in the sense given by Walsh in [16]) obtained as the extension of a centered Gaussian field $\left\{F(\varphi), \varphi \in \mathcal{D}\left(\mathbb{R}_{+} \times \mathbb{R}^{2}\right)\right\}$ with covariance

$$
E(F(\varphi) F(\psi))=\int_{\mathbb{R}_{+}} \mathrm{d} t \int_{\mathbb{R}^{2}} \mathrm{~d} x \int_{\mathbb{R}^{2}} \mathrm{~d} y \varphi(t, x) f(|x-y|) \psi(t, y) .
$$

\footnotetext{
Keywords and phrases: Stochastic partial differential equations, Malliavin Calculus, wave equation, probability densities.

* Partially supported by the grant ERBF MRX CT960075A of the EU.

** Partially supported by the grant BMF 2000-0607 from de Dirección General de Investigación, Ministerio de Ciencia y Tecnologia.

${ }^{1}$ Université Pierre et Marie Curie, Laboratoire de Probabilités, 175/179 rue du Chevaleret, 75013 Paris, France; and Université René Descartes, 45 rue des Saints Pères, 75006 Paris, France; e-mail: mcm@ccr.jussieu.fr

${ }^{2}$ Facultat de Matemàtiques, Universitat de Barcelona, Gran Via 585, 08007 Barcelona, Spain; e-mail: sanz@mat.ub.es
} 
Assume that $f: \mathbb{R}_{+} \rightarrow \mathbb{R}_{+}$is continuous on $] 0, \infty[$. Moreover, we suppose that the measure $\Gamma(\mathrm{d} x)=f(x) \mathrm{d} x$ on $\mathbb{R}^{2}$ is a nonnegative tempered distribution. The initial conditions $u_{0}, v_{0}$, are real functions satisfying some conditions that will be determined later.

We give a meaning to the formal expression (1.2) using its mild formulation, as follows. Let $\mathcal{H}$ be the completion of the inner-product space of measurable functions $\varphi: \mathbb{R}^{2} \rightarrow \mathbb{R}$ such that $\int_{\mathbb{R}^{2}} \mathrm{~d} x \int_{\mathbb{R}^{2}} \mathrm{~d} y|\varphi(x)| f(\mid x-$ $y \mid)|\varphi(y)|<\infty$, endowed with the inner product $\langle\varphi, \psi\rangle_{\mathcal{H}}:=\int_{\mathbb{R}^{2}} \mathrm{~d} x \int_{\mathbb{R}^{2}} \mathrm{~d} y \varphi(x) f(|x-y|) \psi(y)$. Denote by $\left\{e_{j}, j \geq 0\right\}$ a CONS of functions of $\mathcal{H}$. Then $\left\{W_{j}(t)=\int_{0}^{t} \int_{\mathbb{R}^{2}} e_{j}(x) F(\mathrm{~d} s, \mathrm{~d} x), j \geq 0\right\}$ is a sequence of independent standard Brownian motions.

Let $S(t, x),(t, x) \in\left[0, \infty\left[\times \mathbb{R}^{2}\right.\right.$, be the fundamental solution of $\left(\frac{\partial^{2}}{\partial t^{2}}-\Delta\right) g=0$. That is,

$$
S(t, x)=\frac{1}{2 \pi}\left(t^{2}-|x|^{2}\right)^{-1 / 2} 1_{\{|x|<t\}}
$$

Set

$$
X^{0}(t, x)=\int_{\mathbb{R}^{2}} S(t, x-y) v_{0}(y) \mathrm{d} y+\frac{\partial}{\partial t}\left(\int_{\mathbb{R}^{2}} S(t, x-y) u_{0}(y) \mathrm{d} y\right) .
$$

By a solution of equation (1.2) we mean a stochastic process $\left\{u(t, x),(t, x) \in \mathbb{R}_{+} \times \mathbb{R}^{2}\right\}$ satisfying

$$
\begin{aligned}
u(t, x)= & X^{0}(t, x)+\sum_{j \geq 0} \int_{0}^{t}\left\langle S(t-s, x-*) \sigma(u(s, *)), e_{j}(*)\right\rangle_{\mathcal{H}} W_{j}(\mathrm{~d} s) \\
& +\int_{0}^{t} \int_{\mathbb{R}^{2}} S(t-s, x-y) b(u(s, y)) \mathrm{d} s \mathrm{~d} y .
\end{aligned}
$$

In [10] we studied the existence and uniqueness of solution of (1.3) (see also [4]). We also addressed the smoothness of $u(t, x)$, at a fixed point $(t, x) \in \mathbb{R}_{+} \times \mathbb{R}^{2}$, in the sense of Malliavin Calculus, and the existence and smoothness of density for the probability law on $\mathbb{R}^{d}$ of

$$
u(t, \underline{x}):=\left(u\left(t, x_{1}\right), \ldots, u\left(t, x_{d}\right)\right)
$$

with $t>0$ and $x_{1}, \ldots, x_{d}$ distinct points of $\mathbb{R}^{2}$.

This last result has been obtained under the following set of assumptions

(i) there exist $a_{1} \geq a_{2}>0$ such that $2\left(1+a_{2}\right)\left(a_{1}-a_{2}\right)<a_{2} \leq a_{1}<2$, positive constants $C_{1}$ and $C_{2}$ such that for $t \in[0, T]$,

$$
C_{1} t^{a_{1}} \leq \int_{0}^{t} y f(y) \ln \left(1+\frac{t}{y}\right) \mathrm{d} y \leq C_{2} t^{a_{2}}
$$

(ii) $u_{0}: \mathbb{R}^{2} \rightarrow \mathbb{R}$ is of class $\mathcal{C}^{1}$, bounded, with $a_{2} / 2\left(1+a_{2}\right)$-Hölder continuous partial derivatives, $v_{0}: \mathbb{R}^{2} \rightarrow \mathbb{R}$ and there exists $\left.\left.q_{0} \in\right] 4,+\infty\right]$ such that $\left|v_{0}\right|+\left|\nabla u_{0}\right| \in L^{q_{0}}\left(\mathbb{R}^{2}\right)$

(iii) $\sigma$ and $b$ are $\mathcal{C}^{\infty}$ with bounded derivatives of any order $i \geq 1$;

(iv) there exists $a>0$ such that $\left|\sigma\left(u\left(t, x_{j}\right)\right)\right| \geq a$, for any $j=1, \ldots, d$, a.s.

These conditions are satisfied for instance by the function $f(x)=x^{-\alpha}, 0<\alpha<2$, with $a_{1}=a_{2}=2-\alpha$.

In Millet and Morien in [8] there is a slight improvement of the previous result. These authors show that in the above-quoted set of hypothesis, assumptions (i) and (ii) can be replaced by the weaker ones:

(i') there exist $0<a_{2} \leq a_{1}<2$ such that $2\left(a_{1}-a_{2}\right)<a_{2} \wedge 1$, positive constant $C_{1}$ such that for $t \in[0, T]$,

$$
C_{1} t^{a_{1}} \leq \int_{0}^{t} y f(y) \ln \left(1+\frac{t}{y}\right) \mathrm{d} y
$$


and

$$
\int_{0^{+}} y^{1-a_{2}} f(y) \mathrm{d} y<\infty
$$

(ii') $u_{0}: \mathbb{R}^{2} \rightarrow \mathbb{R}$ is of class $\mathcal{C}^{1}$, bounded, with $\frac{1}{2}\left(a_{2} \wedge 1\right)$-Hölder continuous partial derivatives, $\nabla u_{0} \in L^{q_{1}}\left(\mathbb{R}^{2}\right)$ for some $q_{1}>2 ; v_{0}: \mathbb{R}^{2} \rightarrow \mathbb{R}$ belongs to $L^{q_{0}}\left(\mathbb{R}^{2}\right)$ for some $q_{0} \in\left[4 \vee \frac{2}{1-\left(a_{2} \wedge 1\right)}, \infty\right]$.

Recently, in [6] a related problem has been studied. The results apply in particular to equations like (1.2) driven by a centered Gaussian noise with covariance given by

$$
E(F(\varphi) F(\psi))=\int_{\mathbb{R}_{+}} \mathrm{d} t \int_{\mathbb{R}^{2}} \Gamma(\mathrm{d} x)(\varphi(s, .) * \tilde{\psi}(s, .))(x)
$$

where $\Gamma$ is a non-negative, non-negative definite tempered measure, the symbol $*$ means the convolution and $\tilde{\psi}(s, x)=\psi(s,-x)$. Notice that if $\Gamma(\mathrm{d} x)=f(|x|) \mathrm{d} x$ then (1.6) reduces to (1.2). It is proved that for $d=1$ the following set of assumptions yield the existence and smoothness of density for the law of $u(t, x)$, with $t>0$ and $x \in \mathbb{R}^{2}$ : the preceding condition (iii)

(iv') there exists $C>0$ such that $\inf \{|\sigma(z)|, z \in \mathbb{R}\} \geq C$;

(v) there exists $\eta \in\left(0, \frac{3}{4}\right)$ such that

$$
\int_{\mathbb{R}^{2}} \frac{\mu(\mathrm{d} \xi)}{\left(1+|\xi|^{2}\right)^{\eta}}<\infty
$$

where $\mu$ is the spectral measure of $\Gamma$.

In the particular case $\Gamma(\mathrm{d} x)=f(|x|) \mathrm{d} x$ the above condition (1.7) implies property (1.5) of (i') with $a_{2}=$ $2(1-\eta) \in\left(\frac{1}{2}, 2\right)$ (see for instance $\left.[5,15]\right)$.

Denote by $p_{t, \underline{x}}(y)$ the density of $u(t, \underline{x})$ at $y \in \mathbb{R}^{d}$. The purpose of this paper is to prove the next statement.

Theorem 1. Assume that the conditions ( $i$ ', ii', iii) and (iv') hold. Suppose in addition that the functional

$$
J(\varphi, \psi)=\int_{\mathbb{R}^{2}} \mathrm{~d} x \int_{\mathbb{R}^{2}} \mathrm{~d} y \varphi(x) f(|x-y|) \psi(y), \varphi, \psi \in \mathcal{D}\left(\mathbb{R}^{2}\right),
$$

is positive. Then, for any $y \in \mathbb{R}^{d}, p_{t, \underline{x}}(y)$ is stricly positive.

In the remaining of this section we give a brief description of the method we have used to approach this problem.

Let $T>0$ be fixed. The reproducing kernel Hilbert space of the Gaussian process $\left\{\left(W_{j}(t)\right)_{j \geq 0}, t \in[0, T]\right\}$ is the set $H_{T}$ of functions $h:[0, T] \rightarrow \mathbb{R}^{\mathbb{N}}$ such that $\sum_{j \geq 0} \int_{0}^{T}\left|h_{j}(s)\right|^{2} \mathrm{~d} s<\infty$. For any $h \in H_{T}$, let $\left\{\Phi^{h}(t, x),(t, x) \in[0, T] \times \mathbb{R}^{2}\right\}$ be the solution of

$$
\begin{aligned}
\Phi^{h}(t, x)= & X^{0}(t, x)+\sum_{j \geq 0} \int_{0}^{t}\left\langle S(t-s, x-*) \sigma\left(\Phi^{h}(s, *)\right), e_{j}(*)\right\rangle_{\mathcal{H}} h_{j}(s) \mathrm{d} s \\
& +\int_{0}^{t} \int_{\mathbb{R}^{2}} S(t-s, x-y) b\left(\Phi^{h}(s, y)\right) \mathrm{d} s \mathrm{~d} y .
\end{aligned}
$$

This process is called the skeleton of $\left\{u(t, x),(t, x) \in[0, T] \times \mathbb{R}^{2}\right\}$. The function $h \in H_{T} \rightarrow \Phi^{h}(t, x) \in \mathbb{R}$ is Fréchet differentiable (see the Appendix). Set $\Phi^{h}(t, \underline{x})=\left(\Phi^{h}\left(t, x_{1}\right), \ldots, \Phi^{h}\left(t, x_{d}\right)\right)$. Denote by $\gamma_{\Phi^{h}(t, \underline{x})}$ the $d \times d$ matrix whose entries are $\left\langle\bar{D} \Phi^{h}\left(t, x_{i}\right), \bar{D} \Phi^{h}\left(t, x_{j}\right)\right\rangle_{H_{T}}, i, j=1, \ldots, d$, where $\bar{D}$ means the Fréchet derivative operator. It is called the deterministic Malliavin matrix. 
Then we proceed in two steps:

Step 1. Assume (i', ii', iii) and (iv). We prove the equivalence between the next two properties on $y \in \mathbb{R}^{d}:$ (a) $p_{t, \underline{x}}(y)>0$ and (b) there exists $h \in H_{T}$ such that $\Phi^{h}(t, \underline{x})=y$ and $\operatorname{det} \gamma_{\Phi^{h}(t, \underline{x})}>0$.

Step 2. Suppose (i', ii', iii) and (iv'). Then, if the functional $J(\varphi, \psi)$ defined in (1.8) is positive, any $y \in \mathbb{R}^{d}$ satisfies (b).

For diffusion processes satisfying some non-degeneracy requirements the equivalence between the analogue of properties (a, b) above has been proved in [3].

A characterization of the points of positive density, analogous to the equivalence between (a) and (b), for functionals defined in an abstract Wiener space has been developed in [1] and then applied to diffusions.

A similar general setting, more close to the ideas of [3], has been presented in [14]. These abstract formulations allow to analyze many interesting examples in the infinite dimensional case, like solutions of stochastic partial differential equations (see, for instance $[2,9]$ ).

We achieve the goal quoted in Step 1 proving first a weaker (localized) version of the criterium given in [14]. Step 2 requires to solve an inverse problem and also requires a careful analysis of the matrix $\gamma_{\Phi^{h}(t, \underline{x})}$. The positivity of the functional $J(\varphi, \psi)$ is used in the study of the first question, the second one is carried out by exploiting assumption (i').

The programme of the paper is as follows. In Section 2 we precise the tools used in the above-mentioned Step 1. For the proof of $(\mathrm{b}) \Rightarrow(\mathrm{a})$ we use Proposition 4.2.2 in [14]. The proof of $(\mathrm{a}) \Rightarrow(\mathrm{b})$ needs the weaker version of Proposition 4.2.1 in [14] stated as Proposition 2. Section 3 is devoted to complete Step 1. We give an approximation result on a class of evolution equations which includes (1.3) and (1.9). This ensures the validity of the hypothesis needed to apply the criterium established in Section 2; but it has its own interest. Finally, we devote Section 4 to the proof of Step 2.

\section{Points of Positive Density of FunCtionals Defined ON AN ABSTRACT WIENER SPACE}

We devote this section to set up the method of the proof of the first step of Theorem 1 in Section 1 . We follow the approach of [14]; however some modifications are needed.

For the sake of understanding we start by giving some basic notions and facts on Malliavin Calculus and refer the reader to $[13,14]$ for a complete presentation of this topic.

Let $(\Omega, H, P)$ be an abstract Wiener space. For any $h \in H$ we denote be $W(h)$ the Itô-Wiener integral. Let $\mathcal{S}$ be the class of cylindrical Wiener functionals, that is, the set of random vectors of the form

$$
F=f\left(W\left(h_{1}\right), \ldots, W\left(h_{n}\right)\right),
$$

with $f \in \mathcal{C}_{b}^{\infty}\left(\mathbb{R}^{n}\right), h_{1}, \ldots, h_{n} \in H$. For $F$ as in (2.1) the Malliavin derivative is the $H$-valued random variable defined by

$$
D F=\sum_{i=1}^{n} \frac{\partial f}{\partial x_{i}}\left(W\left(h_{1}\right), \ldots, W\left(h_{n}\right)\right) h_{i} .
$$

For any integer $k \geq 1$ and any real number $p \in[1, \infty)$ we define $\mathbb{D}^{k, p}$ as the completion of $\mathcal{S}$ with respect to the norm

$$
\|F\|_{k, p}=\left(E\left(|F|^{p}\right)+\sum_{j=1}^{k} E\left(\left\|D^{j} F\right\|_{H^{\otimes j}}^{p}\right)\right)^{1 / p} .
$$

Suppose that $F=\left(F^{1}, \ldots, F^{d}\right)$ is a random vector whose components belong to $\mathbb{D}^{1,2}$. The Malliavin matrix of $F$ is the $d \times d$ matrix with entries $\left\langle D F^{i}, D F^{j}\right\rangle_{H}, i, j=1, \ldots, d$; it is denoted by $\gamma_{F}$. 
Set $\mathbb{D}^{\infty}=\cap_{k, p} \mathbb{D}^{k, p}$. A random vector $F$ is nondegenerate if $F \in \mathbb{D}^{\infty}\left(\mathbb{R}^{d}\right)$, that means, all the components of $F$ belong to $\mathbb{D}^{\infty}$, and moreover $\operatorname{det}\left(\gamma_{F}\right)^{-1}>0$. It is well-known that the law of a nondegenerate random vector has a $\mathcal{C}^{\infty}$ density with respect to the Lebesgue measure.

Let $\Phi: H \rightarrow \mathbb{R}^{d}$ be Fréchet differentiable. By analogy with the random case we define $\gamma_{\Phi(h)}$ as the matrix with entries $\left\langle\bar{D} \Phi^{i}(h), \bar{D} \Phi^{j}(h)\right\rangle_{H}, \quad i, j=1, \ldots, d$; it is called (after Bismut) the deterministic Malliavin matrix.

For any $y \in \mathbb{R}^{d}$ we consider the following properties:

(A) the density $p$ of the random vector $F$ is strictly positive at $y$;

(B) there exists $h \in H$ with $\Phi(h)=y$ and $\operatorname{det} \gamma_{\Phi(h)}>0$.

For elements $h_{1}, \ldots, h_{d} \in H, z \in \mathbb{R}^{d}$, set $\underline{h}=\left(h_{1}, \ldots, h_{d}\right)$ and define

$$
\left(T_{z} W\right)(h)=W(h)+\sum_{j=1}^{d} z_{j}\left\langle h, h_{j}\right\rangle_{H}, \quad h \in H
$$

Moreover, for $p \in[1, \infty), k \geq 0$, set

$$
R_{\underline{h}, k, p} F=\int_{\{|z| \leq 1\}}\left\|\left(D^{k} F\right)\left(T_{z} W\right)\right\|_{H \otimes k}^{p} \mathrm{~d} z .
$$

We next quote Proposition 4.2.2 of [14].

Proposition 1. Let $F$ be a nondegenerate random vector, $\Phi: H \rightarrow \mathbb{R}^{d}$ be a Fréchet continuously differentiable mapping. Fix $h \in H$ and assume that there exists a sequence of measurable, absolutely continuous transformations $T_{n}^{h}: \Omega \rightarrow \Omega, n \geq 0$, such that, for every $\varepsilon>0, k=0,1,2,3$ and some $p>d$,

$$
\begin{aligned}
& \lim _{n \rightarrow \infty} P\left\{\left|F \circ T_{n}^{h}-\Phi(h)\right|>\varepsilon\right\}=0, \\
& \lim _{n \rightarrow \infty} P\left\{\left\|(D F) \circ T_{n}^{h}-(\bar{D} \Phi)(h)\right\|_{H}>\varepsilon\right\}=0, \\
& \lim _{M \rightarrow \infty} \sup _{n} P\left\{\left(R_{\bar{D} \Phi(h), k, p} F\right) \circ T_{n}^{h}>M\right\}=0 .
\end{aligned}
$$

Then (B) implies (A).

In order to set up the conditions ensuring $(A) \Rightarrow(B)$ we need a localized version of Proposition 4.2.1 in [14]. In fact, the Wiener functional $u(t, \underline{x})$ does not satisfy the convergence assumption needed to apply this proposition. A particular localization on $\Omega$ is required. This leads to a convergence in probability on $\mathbb{D}^{\infty}\left(\mathbb{R}^{d}\right)$.

Let $\left(H_{n}\right)_{n \geq 1}$ be an increasing sequence of finite dimensional subspaces of $H$ such that $\cup_{n \geq 1} H_{n}$ is dense in $H$. Let $W^{n}: \Omega \rightarrow H_{n}$ be a sequence of random variables belonging to $\mathbb{D}^{\infty}$. We introduce a localizing sequence, as follows. Let

$$
A_{n}^{\gamma}=\left\{\omega:\left\|W^{n}(\omega)\right\|_{H}^{2} \leq \gamma C(n)\right\}, \quad n \in \mathbb{N}, \quad \gamma \in(0, \infty)
$$

where $\{C(n), n \geq 1\}$ is an increasing sequence such that

$$
\lim _{n \rightarrow \infty} P\left(\left(A_{n}^{\gamma_{0}}\right)^{c}\right)=0
$$

for some $\gamma_{0}>0$. 
Let $\Psi^{\gamma}: \mathbb{R}_{+} \rightarrow[0,1]$ be a $\mathcal{C}^{\infty}$ function with bounded derivatives of any order, such that

$$
\Psi^{\gamma}(x)=\left\{\begin{array}{lll}
1, & \text { if } & x<\gamma \\
0, & \text { if } & x>2 \gamma
\end{array}\right.
$$

Set $G^{\gamma, n}(\omega)=\Psi^{\gamma}\left(\frac{\left\|W^{n}(\omega)\right\|_{H}^{2}}{C(n)}\right)$. Notice that

$$
\mathbb{1}_{A_{n}^{\gamma}} \leq G^{\gamma, n} \leq \mathbb{1}_{A_{n}^{2 \gamma}} .
$$

Assume that $G^{\gamma, n} \in \mathbb{D}^{\infty}$ uniformly in $n$. That means, for all integer $k \geq 1$ and $p \in[1, \infty)$,

$$
\sup _{n \geq 1}\left\|G^{\gamma, n}\right\|_{k, p}<+\infty \text {. }
$$

Proposition 2. Let $F: \Omega \rightarrow \mathbb{R}^{d}$ be a nondegenerate random vector, $\Phi: H \rightarrow \mathbb{R}^{d}$ be infinitely Fréchet differentiable such that

(H1) $\Phi\left(W^{n}\right) \in \mathbb{D}^{\infty}\left(\mathbb{R}^{d}\right)$, for any $n \geq 1$, and

$$
\lim _{n \rightarrow \infty} E\left(\left\|D^{k}\left(\Phi\left(W^{n}\right)-F\right)\right\|_{H_{T}^{\otimes k}}^{p} \mathbb{1}_{A_{n}^{\gamma}}\right)=0,
$$

for any $k \geq 1, p \in[1, \infty), \gamma \geq \gamma_{0}$.

Then, for each $y \in \mathbb{R}^{d}$, (A) implies (B).

Proof. The arguments are similar to those of Proposition 4.2.1 [14] (see also [3] and [1]) with an additional ingredient of localization.

Let $f$ be any continuous positive function with compact support $\left[a^{0}, b^{0}\right]$ containing $y$; then

$$
c:=\mathbb{E}(f(F))>0 .
$$

Fix $\left.\gamma \geq \gamma_{0}, \varepsilon \in\right] 0, c\left[\right.$; let $n_{0} \in \mathbb{N}$ be such that $\mathbb{P}\left(\left(A_{n}^{\gamma}\right)^{c}\right)<\frac{\varepsilon}{\|f\|_{\infty}}$ for any $n \geq n_{0}$. Then, $0<\mathbb{E}(f(F)) \leq$ $\mathbb{E}\left(f(F) \mathbb{1}_{A_{n}^{\gamma}}\right)+\varepsilon$ and consequently, $\mathbb{E}\left(f(F) \mathbb{1}_{A_{n}^{\gamma}}\right)>0$, for any $n \geq n_{0}$. Therefore (2.6) implies that $\mathbb{E}\left(f(F) G^{\gamma, n}\right)$ $>0$ for $\gamma>\gamma_{0}, n \geq n_{0}$.

For every $M \geq 1$, let $\alpha_{M} \in C_{b}^{\infty}(\mathbb{R})$ be such that $0 \leq \alpha_{M} \leq 1, \alpha_{M}(x)=0$ if $|x| \leq \frac{1}{M}$ and $\alpha_{M}(x)=$ 1 if $|x| \geq \frac{2}{M}$. Since $F$ is nondegenerate we have that $\lim _{M \rightarrow+\infty} \alpha_{M}\left(\operatorname{det} \gamma_{F}\right)=1$, a.s. Consequently, $0<\mathbb{E}\left(f(F) G^{\gamma, n}\right)=\lim _{M \rightarrow+\infty} \mathbb{E}\left(f(F) G^{\gamma, n} \alpha_{M}\left(\operatorname{det} \gamma_{F}\right)\right)$ and there exists a positive integer $M$ such that $\mathbb{E}\left(f(F) G^{\gamma, n} \alpha_{M}\left(\operatorname{det} \gamma_{F}\right)\right)>0$.

We want to prove that for $\gamma \geq \gamma_{0}$

$$
\lim _{n \rightarrow+\infty} \mid \mathbb{E}\left(\left(f(F) \alpha_{M}\left(\operatorname{det} \gamma_{F}\right)-f\left(\Phi\left(W^{n}\right) \alpha_{M}\left(\operatorname{det} \gamma_{\Phi\left(W^{n}\right)}\right)\right) G^{\gamma, n}\right) \mid=0 .\right.
$$

This will imply the existence of a positive integer $n_{0}$ such that

$$
\mathbb{E}\left(f\left(\Phi\left(W^{n}\right)\right) G^{\gamma, n} \alpha_{M}\left(\operatorname{det} \gamma_{\Phi\left(W^{n}\right)}\right)\right)>0,
$$

for any $n \geq n_{0}$. Let $\bar{f}\left(x_{1}, \ldots, x_{d}\right)=\int_{a_{1}^{0}}^{x_{1}} \cdots \int_{a_{d}^{0}}^{x_{d}} f\left(u_{1}, \ldots, u_{d}\right) \mathrm{d} u_{1} \ldots \mathrm{d} u_{d}, a^{0}=\left(a_{1}^{0}, \ldots, a_{d}^{0}\right)$. 
The integration by parts formula of the Malliavin Calculus implies that

$$
\begin{aligned}
T_{n}:=\mathbb{E} & {\left[\left(f(F) \alpha_{M}\left(\operatorname{det} \gamma_{F}\right)-f\left(\Phi\left(W^{n}\right)\right) \alpha_{M}\left(\operatorname{det} \gamma_{\Phi\left(W^{n}\right)}\right)\right) G^{\gamma, n}\right] } \\
=\mathbb{E}[ & \bar{f}(F) H_{\{1, \ldots, d\}}\left(F, G^{\gamma, n} \alpha_{M}\left(\operatorname{det} \gamma_{F}\right)\right) \\
& \left.\quad-\bar{f}\left(\Phi\left(W^{n}\right)\right) H_{\{1, \ldots, d\}}\left(\Phi\left(W^{n}\right), G^{\gamma, n} \alpha_{M}\left(\operatorname{det} \gamma_{\Phi\left(W^{n}\right)}\right)\right)\right]
\end{aligned}
$$

where, for any multiindex $\alpha=\left(\alpha_{1}, \ldots, \alpha_{k}\right) \in\{1, \ldots, d\}^{k}, H_{\alpha}$ are random variables defined recursively as follows. If $U$ is a nondegenerate functional and $V \in \mathbb{D}^{\infty}$ then $H_{\{i\}}(U, V)=\sum_{j=1}^{d} \delta\left(V\left(\gamma_{U}^{-1}\right)^{i j} D U^{j}\right)$ and $H_{\alpha}(U, V)=H_{\left\{\alpha_{k}\right\}}\left(U, H_{\left\{\alpha_{1}, \ldots, \alpha_{k-1}\right\}}(U, V)\right)$, where $\delta$ is the adjoint operator of $D$ which is, as $D$, a local operator. $\delta$ is also called the Skorohod integral.

Since $G^{\gamma, n}=0$ on $\left(A_{n}^{2 \gamma}\right)^{c}$,

$$
\begin{aligned}
& H_{\{1, \ldots, d\}}\left(F, G^{\gamma, n} \alpha_{M}\left(\operatorname{det} \gamma_{F}\right)\right)=\mathbb{1}_{A_{n}^{2 \gamma}} H_{\{1, \ldots, d\}}\left(F, G^{\gamma, n} \alpha_{M}\left(\operatorname{det} \gamma_{F}\right)\right) \\
& \text { and } \\
& H_{\{1, \ldots, d\}}\left(\Phi\left(W^{n}\right), G^{\gamma, n} \alpha_{M}\left(\operatorname{det} \gamma_{\Phi\left(W^{n}\right)}\right)\right)=\mathbb{1}_{A_{n}^{2 \gamma}} H_{\{1, \ldots, d\}}\left(\Phi\left(W^{n}\right), G^{\gamma, n} \alpha_{M}\left(\operatorname{det} \gamma_{\Phi\left(W^{n}\right)}\right)\right) .
\end{aligned}
$$

Consequently $\left|T_{n}\right| \leq T_{n}^{1}+T_{n}^{2}$ with

$$
T_{n}^{1}=\left|\mathbb{E}\left(\left(\bar{f}(F)-\bar{f}\left(\Phi\left(W^{n}\right)\right)\right) \mathbb{1}_{A_{n}^{2 \gamma}} H_{\{1, \ldots, d\}}\left(F, G^{\gamma, n} \alpha_{M}\left(\operatorname{det} \gamma_{F}\right)\right)\right)\right|
$$

and

$$
\begin{aligned}
T_{n}^{2}= & \mid \mathbb{E}\left(\bar{f}\left(\Phi\left(W^{n}\right)\right)\right) \mathbb{1}_{A_{n}^{2 \gamma}}\left[H_{\{1, \ldots, d\}}\left(F, G^{\gamma, n} \alpha_{M}\left(\operatorname{det} \gamma_{F}\right)\right)\right. \\
& \left.-H_{\{1, \ldots, d\}}\left(\Phi\left(W^{n}\right), G^{\gamma, n} \alpha_{M}\left(\operatorname{det} \gamma_{\Phi\left(W^{n}\right)}\right)\right)\right] \mid .
\end{aligned}
$$

Let us first check that $\lim _{n \rightarrow+\infty} T_{n}^{1}=0$. Indeed, $T_{n}^{1} \leq T_{n}^{1,1} \times T_{n}^{1,2}$ where

$$
\begin{aligned}
& T_{n}^{1,1}=\left(\mathbb{E}\left(\left|\bar{f}(F)-\bar{f}\left(\Phi\left(W^{n}\right)\right)\right|^{p} \mathbb{1}_{A_{n}^{2 \gamma}}\right)\right)^{1 / p} \\
& T_{n}^{1,2}=\left\|H_{\{1, \ldots, d\}}\left(F, G^{\gamma, n} \alpha_{M}\left(\operatorname{det} \gamma_{F}\right)\right)\right\|_{L^{q}(\Omega)}
\end{aligned}
$$

with $\frac{1}{p}+\frac{1}{q}=1$.

Assumption (H1) implies that

$$
\varlimsup_{n} T_{n}^{1,1} \leq\|f\|_{\infty} \lim _{n \rightarrow+\infty}\left(\mathbb{E}\left(\left|F-\Phi\left(W^{n}\right)\right|^{p} 1_{A_{n}^{2 \gamma}}\right)\right)^{1 / p}=0 .
$$

Moreover, $\sup _{n} T_{n}^{1,2}<+\infty$. Indeed, Proposition 3.2.2 in [14] yields:

$$
T_{n}^{1,2} \leq C(q, d)\left(\left|\gamma_{F}^{-1}\right|_{k}^{a}\|F\|_{b, c}^{a^{\prime}}\left\|G^{\gamma, n} \alpha_{M}\left(\operatorname{det} \gamma_{F}\right)\right\|_{b^{\prime}, c^{\prime}}^{a^{\prime \prime}}\right)
$$

for some positive real numbers $k, a, c, a^{\prime}, c^{\prime}, a^{\prime \prime}>1$ and positive integers $b, b^{\prime}$. 
The properties on $G^{\gamma, n}$ imply that $\sup _{n} T_{n}^{1,2}<+\infty$. We now prove that $\lim _{n \rightarrow+\infty} T_{n}^{2}=0$. For any $p>1$,

$$
\begin{aligned}
T_{n}^{2} \leq & \|\bar{f}\|_{\infty} \mathbb{E} \mid \mathbb{1}_{A_{n}^{2 \gamma}\left[H_{\{1, \ldots, d\}}\left(F, G^{\gamma, n} \alpha_{M}\left(\operatorname{det} \gamma_{F}\right)\right)\right.} \\
& \left.-H_{\{1, \ldots, d\}}\left(\Phi\left(W^{n}\right), G^{\gamma, n} \alpha_{M}\left(\operatorname{det} \gamma_{\Phi\left(W^{n}\right)}\right)\right)\right] \mid
\end{aligned}
$$

The $L^{p}$-inequalities for the Skorohod integral yield

$$
T_{n}^{2} \leq C(d)\|\bar{f}\|_{\infty} \mathbb{E}\left|\mathbb{1}_{A_{n}^{2 \gamma}}\left(\left\|F-\Phi\left(W^{n}\right)\right\|_{e, r}+\left\|G^{\gamma, n}\left(\alpha_{M}\left(\operatorname{det} \gamma_{F}\right)\right)-\alpha_{M}\left(\operatorname{det} \gamma_{\Phi\left(W^{n}\right)}\right)\right\|_{e^{\prime}, r^{\prime}}\right)\right|
$$

for some $r, r^{\prime}>1, e, e^{\prime} \in \mathbb{N}$ (see [12] for the one dimensional case).

Thus $\lim _{n \rightarrow \infty} T_{n}^{2}=0$. This finishes the proof of (2.8) and therefore that of (2.9).

Next we consider a function $\beta_{K}: \mathbb{R} \rightarrow[0,1], \mathcal{C}^{\infty}$, such that $\beta_{K}(x)=1$ if $|x| \leq K$ and $\beta_{K}(x)=0$ if $|x| \leq K+1$. Since $\left\|W^{n}\right\|_{H}^{2}$ is finite a.s., it is clear that for any fixed $n \geq 1, \beta_{K}\left(\left\|W^{n}\right\|_{H}^{2}\right)$ converges to 1 a.s. as $K \rightarrow \infty$.

Notice that since the functions $f$ and $\alpha_{M}$ are positive and the random variable $G^{\gamma, n}$ is positive and bounded by 1 , the inequality (2.9) implies that for any $n \geq n_{0}$,

$$
\mathbb{E}\left(f\left(\Phi\left(W^{n}\right)\right) \alpha_{M}\left(\operatorname{det} \gamma_{\Phi\left(W^{n}\right)}\right)\right)>0
$$

Thus, for any fixed $n \geq n_{0}$ there exists $K_{0}(n)$ such that for any $K \geq K_{0}(n)$,

$$
\mathbb{E}\left(f\left(\Phi\left(W^{n}\right)\right) \alpha_{M}\left(\operatorname{det} \gamma_{\Phi\left(W^{n}\right)}\right) \beta_{K}\left(\left\|W^{n}\right\|_{H}^{2}\right)\right)>0 .
$$

This yields, for any $K \geq K_{0}(n)$ and any $\varepsilon>0$

$$
\mathbb{P}\left(\left|\Phi\left(W^{n}\right)-y\right|<\varepsilon,\left|\operatorname{det} \gamma_{\Phi\left(W^{n}\right)}\right| \geq \frac{1}{M},\left\|W^{n}\right\|_{H}^{2} \leq K+1\right)>0 .
$$

Indeed, otherwise, if for some $K \geq K_{0}(n)$ and some $\varepsilon>0$

$$
\mathbb{P}\left(\left|\Phi\left(W^{n}\right)-y\right|<\varepsilon,\left|\operatorname{det} \gamma_{\Phi\left(W^{n}\right)}\right| \geq \frac{1}{M},\left\|W^{n}\right\|_{H}^{2} \leq K+1\right)=0,
$$

one could find a function $f$ bounded, positive and continuous such that $y \in \operatorname{supp} f$ and

$$
\mathbb{E}\left(f\left(\Phi\left(W^{n}\right)\right) \alpha_{M}\left(\operatorname{det} \gamma_{\Phi\left(W^{n}\right)}\right) \beta_{K}\left(\left\|W^{n}\right\|_{H}^{2}\right)\right)=0
$$

because $\mathbb{1}_{\left\{\left|\operatorname{det} \gamma_{\Phi\left(W^{n}\right)}\right| \geq \frac{1}{M}\right\}} \geq \alpha_{M}\left(\operatorname{det} \gamma_{\Phi\left(W^{n}\right)}\right)$ and $\mathbb{1}_{\left\{\left\|W^{n}\right\|_{H}^{2} \leq K+1\right\}} \geq \beta_{K}\left(\left\|W^{n}\right\|_{H}^{2}\right)$. This contradicts (2.11). Let $\bar{k}=K_{0}(n)+1$. Then from $(2.12)$ we can find a sequence of elements $h_{m} \in H_{n}$ such that for any $m \geq 1$, $\left|\Phi\left(h_{m}\right)-y\right|<\frac{1}{m},\left\|h_{m}\right\|_{H}^{2} \leq \bar{k}$ and $\left|\operatorname{det} \gamma_{\Phi\left(h_{m}\right)}\right| \geq \frac{1}{M}$.

The compactness of bounded and closed sets in $H_{n}$ implies that we can select a subsequence converging to some element $h \in H$ which verifies $\Phi(h)=y$ and $\left|\operatorname{det} \gamma_{\Phi(h)}\right|>0$. That means (B) holds.

In this article we shall apply the preceding results to the following particular case. Fix any $T>0$. Consider a sequence $\left\{W_{j}(t), t \in[0, T]\right\}, j \geq 0$, of standard Wiener processes and let $\left(\Omega, H_{T}, P\right)$ be the associated Wiener space. That means $\Omega=\mathcal{C}\left([0, T] ; \mathbb{R}^{\mathbb{N}}\right), H_{T}$ is the Hilbert space $L^{2}\left([0, T] ; \mathbb{R}^{\mathbb{N}}\right)$ and $P$ is the Wiener measure on $\Omega$. The random vector $F$ will be $u(t, \underline{x})=\left(u\left(t, x_{1}\right), \ldots, u\left(t, x_{d}\right)\right)$, the solution to the wave equation (1.3) at different points $\left(t, x_{1}\right), \ldots,\left(t, x_{d}\right)$ of $[0, \bar{T}] \times \mathbb{R}^{2}$. The functional $\Phi: H \rightarrow \mathbb{R}^{d}$ will be the skeleton $\Phi^{h}(t, \underline{x})=$ $\left(\Phi^{h}\left(t, x_{1}\right), \ldots, \Phi^{h}\left(t, x_{d}\right)\right)$ defined in $(1.9)$.

Let us precise which are the sequences $\left(T_{n}^{h}\right)_{n \geq 0}$ and $\left(W^{n}\right)_{n \geq 0}$ in the above Propositions 1 and 2. 
For any fixed $n \in \mathbb{N}$ we denote by $\Delta_{i}$ the interval $\left[\frac{i T}{2^{n}}, \frac{(i+1) T)}{2^{n}}\right)$ and write $W_{j}\left(\Delta_{i}\right)$ for the increment $W_{j}\left(\frac{(i+1) T}{2^{n}}\right)-W_{j}\left(\frac{i T}{2^{n}}\right)$.

Let $W^{n}=\left(W_{j}^{n}=\int_{0} \dot{W}_{j}^{n}(s) \mathrm{d} s, j \in \mathbb{N}\right)$ be as in [11], that is, $\dot{W}_{j}^{n}=0$ if $j>n$ and, for $0 \leq j \leq n$,

$$
\dot{W}_{j}^{n}(t)= \begin{cases}\sum_{i=1}^{2^{n}} 2^{n} T^{-1} W_{j}\left(\Delta_{i-1}\right) 1_{\Delta_{i}}(t), & \text { if } t \in\left[2^{-n} T, T\right], \\ 0, & \text { if } t \in\left[0,2^{-n} T\right] .\end{cases}
$$

Notice that for each $n \in \mathbb{N},\left(\dot{W}_{j}^{n}, 0 \leq j \leq n\right)$ belongs to the finite dimensional subspace of $H_{T}$ generated by $\left\{2^{n} T^{-1} 1_{\Delta_{i}}, i=1, \ldots, 2^{n}-1\right\}$. We identify $\left(\dot{W}_{j}^{n}, 0 \leq j \leq n\right)$ with an element of $H_{T}$ by putting $\dot{W}_{j}^{n} \equiv 0$ for $j>n$.

For any $h \in H_{T}$ define

$$
T_{n}^{h}(\omega)=\left(W_{j}-W_{j}^{n}+\int_{0}^{\cdot} h_{j}(s) \mathrm{d} s, j \geq 0\right) .
$$

Girsanov's theorem yields that $P \circ\left(T_{n}^{h}\right)^{-1} \ll P$.

Finally, the localizing sequence $A_{n}^{\gamma}, n \geq 1$ of Proposition 2 is defined as follows. Fix $\gamma>2 T \ln 2, t \in[0, T]$; then

$$
A_{n}^{\gamma}(t)=\left\{\left\|W^{n} \mathbb{1}_{[0, t]}\right\|_{H_{T}}^{2} \leq \gamma n^{2} 2^{n} T^{-1}\right\}
$$

Clearly the sets $A_{n}^{\gamma}(t)$ decrease in $t$ and increase in $\gamma$. Moreover,

$$
\lim _{n \rightarrow \infty} P\left(A_{n}^{\gamma}(T)^{c}\right)=0
$$

Indeed,

$$
\begin{aligned}
& P\left(A_{n}^{\gamma}(T)^{c}\right) \leq \sum_{j=1}^{n} \sum_{i=0}^{2^{n}-1} P\left(\left|W_{j}\left(\Delta_{i}\right)\right|^{2} \geq \gamma n 2^{-n}\right) \\
& \leq n 2^{n} P\left(|Z| \geq \sqrt{\gamma} n^{\frac{1}{2}} T^{-\frac{1}{2}}\right) \leq \frac{n 2^{n} T^{\frac{1}{2}}}{\sqrt{\gamma} n^{\frac{1}{2}}} \exp \left(-\frac{\gamma n}{2 T}\right) \\
& \leq \gamma^{-\frac{1}{2}} n^{\frac{1}{2}} T^{\frac{1}{2}} \exp \left(-n\left(\frac{\gamma}{2 T}-\log 2\right)\right)
\end{aligned}
$$

where $Z$ is a standard Gaussian random variable. Thus (2.17) holds true.

A simple computation shows that for any $0 \leq t<t^{\prime} \leq T, p \in[2, \infty)$ and $\left[t, t^{\prime}\right] \subset \Delta_{i}$ for some $i$,

$$
E\left(\left\|W^{n} \mathbb{1}_{\left[t, t^{\prime}\right]}\right\|_{H_{T}}^{p} \leq n^{\frac{p}{2}}\right.
$$


In fact,

$$
\begin{aligned}
& E\left(\left\|W^{n} 1_{\left[t, t^{\prime}\right]}\right\|_{H_{T}}^{p}\right)=E\left(\left(\sum_{j=1}^{n} 2^{2 n} T^{-2}\left(W_{j}\left(\Delta_{i-1}\right)\right)^{2}\left(t-t^{\prime}\right)\right)^{\frac{p}{2}}\right) \\
& \leq 2^{n p} T^{-p}\left(t-t^{\prime}\right)^{\frac{p}{2}} E\left(\sum_{j=1}^{n}\left(W_{j}\left(\Delta_{i-1}\right)\right)^{2}\right)^{\frac{p}{2}} \\
& \leq n^{\frac{p}{2}} .
\end{aligned}
$$

Let

$$
G^{\gamma, n}(\omega)=\Psi^{\gamma}\left(\frac{\left\|W^{n}\right\|_{H_{T}}^{2}}{n^{2} 2^{n} T^{-1}}\right)=\Psi^{\gamma}\left(n^{-2} \sum_{j=1}^{n} \sum_{i=1}^{2^{n}} W_{j}\left(\Delta_{i-1}\right)^{2}\right)
$$

$n \geq 1$, where $\Psi^{\gamma}$ is given by (2.5). This sequence satisfies property (2.7). Indeed, this can be proved by direct computations, as follows.

Let $k \in \mathbb{N}$ and fix a set $B_{k}=\left\{\alpha_{i}=\left(r_{i}, j_{i}\right) \in \mathbb{R}_{+} \times \mathbb{N}, i=1, \ldots k\right\}$. Let $\alpha=\left(\alpha_{1}, \ldots, \alpha_{k}\right)$ and denote by $P_{m}$ the set of partitions of $B_{k}$ consisting of $m$ disjoint subsets $p_{1}, \ldots, p_{m}, m=1, \ldots, k$; set $\left|p_{i}\right|=$ card $p_{i}$. Let $Y$ be any random variable in $\mathbb{D}^{k, 2}, k \geq 1, g$ be a real $\mathcal{C}^{k}$ function with bounded derivatives up to order $k$. Leibniz's rule for Malliavin's derivatives yields

$$
D_{\alpha}^{k}(g(Y))=\sum_{m=1}^{k} \sum_{P_{m}} c_{m} g^{(m)}(Y) \prod_{i=1}^{m} D_{p_{i}}^{\left|p_{i}\right|} Y
$$

with some positive coefficients $c_{m}, m=1, \ldots, k, c_{1}=1$. We want to apply this formula to $g=\Psi^{\gamma}$ and $Y=n^{-2} \sum_{j=1}^{n} \sum_{i=1}^{2^{n}} W_{j}\left(\Delta_{i-1}\right)^{2}, n \geq 1$. Notice that these random variables have null components on the $n$-th Wiener chaos for $n \geq 3$. Hence, it suffices to prove (2.7) for $k=0,1,2$ and $p \in[1, \infty)$. For these values of $k$, the order of the derivatives in the right hand-side of (2.20) are clearly less or equal to 2.

For $k=0$, the result is obvious, since $\Psi^{\gamma}$ is bounded by 1 . Set

$$
F_{n}=n^{-2} \sum_{j=1}^{n} \sum_{i=1}^{2^{n}} W_{j}\left(\Delta_{i-1}\right)^{2}
$$

then, $D_{r, j} F_{n}=0$, if $j>n$ and for $j \leq n$

$$
D_{r, j} F_{n}=n^{-2} \sum_{i=1}^{2^{n}} 2 W_{j}\left(\Delta_{i-1}\right) \mathbb{1}_{\Delta_{i-1}}(r)
$$

Furthermore, $D_{\left(r_{1}, j_{1}\right)\left(r_{2}, j_{2}\right)}^{2} F_{n}=0$, if $j_{1}>n$ or $j_{1} \leq n$ but $j_{1} \neq j_{2}$ and, for $j_{1}=j_{2} \leq n$,

$$
D_{\left(r_{1}, j_{1}\right)\left(r_{2}, j_{2}\right)}^{2} F_{n}=n^{-2} \sum_{i=1}^{2^{n}} 2 \mathbb{1}_{\Delta_{i-1}}\left(r_{1}\right) \mathbb{1}_{\Delta_{i-1}}\left(r_{2}\right)
$$


Applying Hölder's inequality it is easy to check that

$$
E\left(\sum_{j \in \mathbb{N}} \int_{0}^{T} \mathrm{~d} r\left|D_{r, j} F_{n}\right|^{2}\right)^{\frac{p}{2}} \leq C n^{-\frac{3}{2} p} 2^{p\left(-\frac{n}{2}+1\right)}
$$

Moreover, a direct computation shows that

$$
E\left(\sum_{j_{1}, j_{2} \in \mathbb{N}} \int_{0}^{T} \mathrm{~d} r_{1} \int_{0}^{T} \mathrm{~d} r_{2}\left|D_{\left(r_{1}, j_{1}\right)\left(r_{2}, j_{2}\right)}^{2} F_{n}\right|^{2}\right)^{\frac{p}{2}} \leq C n^{-\frac{3}{2} p 2^{p\left(-\frac{n}{2}+1\right)}}
$$

Thus, for any $p \in[1, \infty)$ and $k=0,1,2$, we have that $\sup _{n}\left\|F_{n}\right\|_{k, p}<\infty$. Then, by means of (2.20) we complete the proof of (2.7) for the sequence of random variables defined in (2.19).

\section{Approximation in probability in $\mathbb{D}^{\infty}$. Characterization of POINTS OF POSITIVE DENSity}

In this section we present an approximation result for a class of evolution equations which include as particular cases (1.3) and (1.9). This general setting allows to check the validity of the assumptions of Propositions 1 and 2 for the Wiener functional $F=u(t, \underline{x})$.

Let $A, B, G, b: \mathbb{R} \rightarrow \mathbb{R}, h \in H_{T}$. Consider the evolution equations

$$
\begin{aligned}
X_{n}(t, x)= & X^{0}(t, x)+\sum_{j \geq 0} \int_{0}^{t}\left\{\left\langle S(t-s, x-*) A\left(X_{n}(s, *)\right), e_{j}(*)\right\rangle_{\mathcal{H}} W_{j}(\mathrm{~d} s)\right. \\
& +\left\langle S(t-s, x-*) B\left(X_{n}(s, *)\right), e_{j}(*)\right\rangle_{\mathcal{H}} \dot{W}_{j}^{n}(s) \mathrm{d} s \\
& \left.+\left\langle S(t-s, x-*) G\left(X_{n}(s, *)\right), e_{j}(*)\right\rangle_{\mathcal{H}} h_{j}(s) \mathrm{d} s\right\} \\
& +\int_{0}^{t} \int_{\mathbb{R}^{2}} S(t-s, x-y) b\left(X_{n}(s, y)\right) \mathrm{d} s \mathrm{~d} y, \\
X(t, x)=\quad & X^{0}(t, x)+\sum_{j \geq 0} \int_{0}^{t}\left\{\left\langle S(t-s, x-*)(A+B)(X(s, *)), e_{j}(*)\right\rangle_{\mathcal{H}} W_{j}(\mathrm{~d} s)\right. \\
+ & \left.\left\langle S(t-s, x-*) G(X(s, *)), e_{j}(*)\right\rangle_{\mathcal{H}} h_{j}(s) \mathrm{d} s\right\} \\
+ & \int_{0}^{t} \int_{\mathbb{R}^{2}} S(t-s, x-y) b(X(s, y)) \mathrm{d} s \mathrm{~d} y .
\end{aligned}
$$

The existence and uniqueness of solution for equations (3.1) and (3.2) have been addressed in [11]. Notice that the processes $X_{n}$ and $X$ depend on $h \in H_{T}$.

We introduce the following set of assumptions (see (1.5) and (ii') in Sect. 1).

There exists $\left.\beta_{0} \in\right] 0,2[$ such that

(C1) $\int_{0^{+}} r^{1-\beta_{0}} f(r) \mathrm{d} r<+\infty$;

(C2) $u_{0}: \mathbb{R}^{2} \rightarrow \mathbb{R}$ is of class $\mathcal{C}^{1}$, bounded, with $\frac{1}{2}\left(\beta_{0} \wedge 1\right)$-Hölder continuous partial derivatives, $\nabla u_{0} \in L^{q_{1}}\left(\mathbb{R}^{2}\right)$ for some $q_{1}>2 ; v_{0}: \mathbb{R}^{2} \rightarrow \mathbb{R}$ belongs to $L^{q_{0}}\left(\mathbb{R}^{2}\right)$ for some $\left.\left.q_{0} \in\right] 4 \vee \frac{2}{1-\left(\beta_{0} \wedge 1\right)}, \infty\right]$;

(C3) the coefficients $A, B, G, b$ of equation (3.1) (and (3.2)) are $\mathcal{C}^{\infty}$ functions with bounded derivatives of any order $k \geq 1$. 
Conditions (C1-C3) ensure that the trajectories of the solution of (1.3) are $\beta$-Hölder continuous in $(t, x)$ for $\beta<\frac{1}{2}\left(\beta_{0} \wedge 1\right)$ (see Th. 2.2 in [8] and Prop. 1.4 in [10]).

For any separable Hilbert space $\mathbb{E}$ we denote by $H_{T}(\mathbb{E})$ the Hilbert space of functions $g:[0, T] \rightarrow \mathbb{E}^{\mathbb{N}}$ such that $\sum_{j \geq 0} \int_{0}^{T}\left\|g_{j}(s)\right\|_{\mathbb{E}}^{2} \mathrm{~d} s<\infty$. Notice that $H_{T}(\mathbb{R})=H_{T}$. In this section we will deal with $\mathbb{E}=\mathbb{R}^{d}$ and $\mathbb{E}=\mathcal{H}$, where $\mathcal{H}$ has been defined in the introduction.

We now state the main technical result of this section. We shall see later in Theorem 3 that by a particular choice of the coefficients $A, B, G$ of equations (3.1) and (3.2), the next Theorem 2 allows to complete the first step of our programme.

Theorem 2. Assume (C1-C3). Then, for any $p \in(1, \infty), k \in \mathbb{Z}_{+}, \gamma>2 T$ ln 2 and every compact set $K \subset \mathbb{R}^{2}$,

$$
\lim _{n \rightarrow \infty} \sup _{0 \leq t \leq T} \sup _{\underline{x} \in K^{d}} E\left(\left\|D^{k}\left(X_{n}(t, \underline{x})-X(t, \underline{x})\right)\right\|_{H_{T}\left(\mathbb{R}^{d}\right) \otimes k}^{p} 1_{A_{n}^{\gamma}(t)}\right)=0
$$

where, for $k=0, D^{0}=I d$ and $\|\cdot\|_{H_{T}\left(\mathbb{R}^{d}\right) \otimes 0}$ is the Euclidean norm in $\mathbb{R}^{d}$. Moreover, the convergence in (3.3) is uniform in $h$ on bounded sets of $H_{T}$.

This theorem provides an extension of Proposition 2.3 in [11], where the convergence stated in (3.3) has been proved in the $L^{p}$ norm. Actually, in this proposition the localizing sequence is given by $\bar{A}_{n}^{\gamma}(t)=$ $\left\{\sup _{0 \leq j \leq n} \sup _{0 \leq i \leq\left(\left[2^{n} t T^{-1}\right]-1\right)^{+}}\left|W_{j}\left(\Delta_{i}\right)\right| \leq \sqrt{\gamma} 2^{-n / 2} n^{1 / 2}\right\}$, which is included in $A_{n}^{\gamma}(t)$ and also satisfies $\lim _{n \rightarrow \infty} P\left(\left(\bar{A}_{n}^{\gamma}(T)\right)^{c}\right)=0$ (see Lem. 2.1 in [11]). However looking carefully at the proof we realize that only two facts concerning the localization are needed: (a) $E\left(\left\|W^{n}\right\|_{H_{T}}^{p} \mathbb{1}_{\bar{A}_{n}^{\gamma}(t)}\right) \leq C n^{p} 2^{\frac{n p}{2}}$ and (b) $E\left(\left\|W^{n} \mathbb{1}_{\left[t_{n}, t\right]}\right\|_{H_{T}}^{p} \mathbb{1}_{\bar{A}_{n}^{\gamma}(t)}\right) \leq$ $C n^{p}$. Here we have used the following notation: for any $n \geq 1, t \in[0, T]$, we set $\underline{t}_{n}=\max \left\{k 2^{-n} T ; k=\right.$ $\left.1, \ldots, 2^{n}-1: k 2^{-n} T \leq t\right\}, t_{n}=\left(\underline{t}_{n}-2^{-n} T\right) \vee 0$. Property (a) is clearly true with $A_{n}^{\gamma}(t)$ instead of $\bar{A}_{n}^{\gamma}(t)$, by the very definition of $A_{n}^{\gamma}(t)$. Property (b) is a trivial consequence of (2.18).

The proof in the $\mathbb{D}^{\infty}$ convergence consists in a quite long and tricky exercise with almost no new ideas. For this reason we do not give a detailed proof. Instead, we draw an outline and also state the technical lemmas which are needed. With these ingredients we provide the readers interested in a complete proof with the main guidelines to check by themselves this extension.

Let us introduce some additional notation.

$$
\begin{aligned}
X_{n}^{-}(t, x)= & X^{0}(t, x)+\sum_{j \geq 0} \int_{0}^{t_{n}}\left\{\left\langle S(t-s, x-*) A\left(X_{n}(s, *)\right), e_{j}(*)\right\rangle_{\mathcal{H}} W_{j}(\mathrm{~d} s)\right. \\
& +\left\langle S(t-s, x-*) B\left(X_{n}(s, *)\right), e_{j}(*)\right\rangle_{\mathcal{H}} \dot{W}_{j}^{n}(s) \mathrm{d} s \\
& \left.+\left\langle S(t-s, x-*) G\left(X_{n}(s, *)\right), e_{j}(*)\right\rangle_{\mathcal{H}} h_{j}(s) \mathrm{d} s\right\} \\
& +\int_{0}^{t_{n}} \int_{\mathbb{R}^{2}} S(t-s, x-y) b\left(X_{n}(s, y)\right) \mathrm{d} s \mathrm{~d} y, \\
X^{-}(t, x)= & X^{0}(t, x)+\sum_{j \geq 0} \int_{0}^{t_{n}}\left\{\left\langle S(t-s, x-*)(A+B)(X(s, *)), e_{j}(*)\right\rangle_{\mathcal{H}} W_{j}(\mathrm{~d} s)\right. \\
+ & \left.\left\langle S(t-s, x-*) G(X(s, *)), e_{j}(*)\right\rangle_{\mathcal{H}} h_{j}(s) \mathrm{d} s\right\} \\
& +\int_{0}^{t_{n}} \int_{\mathbb{R}^{2}} S(t-s, x-y) b(X(s, y)) \mathrm{d} s \mathrm{~d} y .
\end{aligned}
$$

Notice that, although it is not explicit in the notation, $X^{-}$depends on $n$. 
In Section 2 of [10] we have proved that, if $G=0, X(t, x) \in \mathbb{D}^{\infty}$, for any $t \in[0, T], x \in \mathbb{R}^{2}$. A slight modification of the proof allows to establish the same result for $G$ satisfying the assumptions of Theorem 2 as well as for $X_{n}(t, x), X_{n}^{-}(t, x)$ and $X^{-}(t, x)$, respectively. Moreover, for any $k \in \mathbb{Z}_{+}$and $p \in[1, \infty)$,

$$
\sup _{(t, x) \in[0, T] \times \mathbb{R}^{2}}\left\|D^{k} X(t, x)\right\|_{L^{p}\left(\Omega ; H_{T}^{\otimes k}\right)}<\infty
$$

We also need the explicit form of the evolution equations satisfied by the Malliavin derivatives of these processes. To this end we consider the chain rule given in $(2.20)$. Let $\Delta_{\alpha}(g, Y)=D_{\alpha}^{k}(g(Y))-g^{\prime}(Y) D_{\alpha}^{k} Y$ and $\Delta(g, Y)$ be the stochastic process with components $\Delta_{\alpha}(g, Y)$. For any $r_{1}, \cdots, r_{k} \in \mathbb{R}$ we define $\bigvee_{i} r_{i}=\max \left(r_{1}, \cdots, r_{k}\right)$ and for $\alpha_{i}=\left(r_{i}, j_{i}\right) \in \mathbb{R}_{+} \times \mathbb{N}, \hat{\alpha}_{i}=\left(\alpha_{1}, \ldots, \alpha_{i-1}, \alpha_{i+1}, \ldots, \alpha_{k}\right)$.

Then, following the proof of Theorem 2.2 in [10], we obtain

$$
\begin{aligned}
& D_{\alpha}^{k} X_{n}(t, x)=\sum_{i=1}^{k}\left\langle S\left(t-r_{i}, x-*\right) D_{\hat{\alpha}_{i}}^{k-1}\left(A\left(X_{n}\left(r_{i}, *\right)\right)\right), e_{j_{i}}(*)\right\rangle_{\mathcal{H}} \\
& +\sum_{j \geq 0} \int_{\bigvee_{i} r_{i}}^{t}\left\langle S(t-s, x-*) \Delta_{\alpha}\left(A, X_{n}(s, *)\right), e_{j}(*)\right\rangle_{\mathcal{H}} W_{j}(\mathrm{~d} s) \\
& +\sum_{i=1}^{k} \int_{\bigvee_{i} r_{i}}^{t}\left\langle S(t-s, x-*) D_{\hat{\alpha}_{i}}^{k-1}\left(B\left(X_{n}(s, *)\right)\right), e_{j_{i}}(*)\right\rangle_{\mathcal{H}} \\
& \times \sum_{i=1}^{2^{n}} 2^{n} T^{-1} \times 1_{\Delta_{i-1}}\left(r_{i}\right) \times 1_{\Delta_{i}}(s) \mathrm{d} s \\
& +\sum_{j=0}^{n} \int_{\bigvee_{i} r_{i}}^{t}\left\langle S(t-s, x-*) \Delta_{\alpha}\left(B, X_{n}(s, *)\right), e_{j}(*)\right\rangle_{\mathcal{H}} \dot{W}_{j}^{n}(s) \mathrm{d} s \\
& +\sum_{j \geq 0} \int_{\bigvee_{i} r_{i}}^{t}\left\langle S(t-s, x-*) \Delta_{\alpha}\left(G, X_{n}(s, *)\right), e_{j}(*)\right\rangle_{\mathcal{H}} h_{j}(s) \mathrm{d} s \\
& +\int_{\bigvee_{i} r_{i}}^{t} \int_{\mathbb{R}^{2}} S(t-s, x-y) \Delta_{\alpha}\left(b, X_{n}(s, y)\right) \mathrm{d} s \mathrm{~d} y \\
& +\sum_{j \geq 0} \int_{\bigvee_{i} r_{i}}^{t}\left\langle S(t-s, x-*) A^{\prime}\left(X_{n}(s, *)\right) D_{\alpha}^{k} X_{n}(s, *), e_{j}(*)\right\rangle_{\mathcal{H}} W_{j}(\mathrm{~d} s) \\
& +\sum_{j=0}^{n} \int_{\bigvee_{i} r_{i}}^{t}\left\langle S(t-s, x-*) B^{\prime}\left(X_{n}(s, *)\right) D_{\alpha}^{k} X_{n}(s, *), e_{j}(*)\right\rangle_{\mathcal{H}} \dot{W}_{j}^{n}(s) \mathrm{d} s \\
& +\sum_{j \geq 0} \int_{\bigvee_{i} r_{i}}^{t}\left\langle S(t-s, x-*) G^{\prime}\left(X_{n}(s, *)\right) D_{\alpha}^{k} X_{n}(s, *), e_{j}(*)\right\rangle_{\mathcal{H}} h_{j}(s) \mathrm{d} s \\
& +\int_{\bigvee_{i} r_{i}}^{t} \int_{\mathbb{R}^{2}} S(t-s, x-y) b^{\prime}\left(X_{n}(s, y)\right) D_{\alpha}^{k} X_{n}(s, y) \mathrm{d} s \mathrm{~d} y
\end{aligned}
$$


and similarly,

$$
\begin{aligned}
D_{\alpha}^{k} X(t, x)= & \sum_{i=1}^{k}\left\langle S\left(t-r_{i}, x-*\right) D_{\hat{\alpha}_{i}}^{k-1}\left((A+B)\left(X\left(r_{i}, *\right)\right)\right), e_{j_{i}}(*)\right\rangle_{\mathcal{H}} \\
& +\sum_{j \geq 0} \int_{\bigvee_{i} r_{i}}^{t}\left\langle S(t-s, x-*) \Delta_{\alpha}(A+B, X(s, *)), e_{j}(*)\right\rangle_{\mathcal{H}} W_{j}(\mathrm{~d} s) \\
& +\sum_{j \geq 0} \int_{\bigvee_{i} r_{i}}^{t}\left\langle S(t-s, x-*) \Delta_{\alpha}(G, X(s, *)), e_{j}(*)\right\rangle_{\mathcal{H}} h_{j}(s) \mathrm{d} s \\
& +\int_{\bigvee_{i} r_{i}}^{t} \int_{\mathbb{R}^{2}} S(t-s, x-y) \Delta_{\alpha}(b, X(s, y)) \mathrm{d} s \mathrm{~d} y \\
& +\sum_{j \geq 0} \int_{\bigvee_{i} r_{i}}^{t}\left\langle S(t-s, x-*)(A+B)^{\prime}(X(s, *)) D_{\alpha}^{k} X(s, *), e_{j}(*)\right\rangle_{\mathcal{H}} W_{j}(\mathrm{~d} s) \\
& +\sum_{j \geq 0} \int_{\bigvee_{i} r_{i}}^{t}\left\langle S(t-s, x-*) G^{\prime}(X(s, *)) D_{\alpha}^{k} X(s, *), e_{j}(*)\right\rangle_{\mathcal{H}} h_{j}(s) \mathrm{d} s \\
& +\int_{\bigvee_{i} r_{i}}^{t} \int_{\mathbb{R}^{2}} S(t-s, x-y) b^{\prime}(X(s, y)) D_{\alpha}^{k} X(s, y) \mathrm{d} s \mathrm{~d} y,
\end{aligned}
$$

in the case where $\bigvee r_{i} \leq t$. Otherwise, $D_{\alpha}^{k} X_{n}(t, x)=D_{\alpha}^{k} X(t, x)=0$.

The equations satisfied by $D_{\alpha}^{k} X_{n}^{-}(t, x)$ (resp. $\left.D_{\alpha}^{k} X^{-}(t, x)\right)$ are obtained substituting in (3.7) (resp. in (3.8)) the upper bound in the integral by $t_{n}$ and multiplying the first term of the right hand-side of (3.7) (resp. of (3.8)) by $1_{\left[0, t_{n}\right]}\left(r_{i}\right)$.

Outline of the proof of Theorem 2. We shall apply induction on $k$ and assume that $d=1$. The proof for $d>1$ is analogous. For $k=0$, the convergence (3.3) has been proved in Proposition 2.3 of [11].

Set

$$
D_{\alpha}^{k}\left(X_{n}(t, x)-X(t, x)\right)=\sum_{i=1}^{8} V_{i, \alpha}^{n}(t, x)
$$

where

$$
\begin{aligned}
& V_{1, \alpha}^{n}(t, x)=\sum_{i=1}^{k}\left\langle S\left(t-r_{i}, x-*\right)\left[D_{\hat{\alpha}_{i}}^{k-1}\left(A\left(X_{n}\left(r_{i}, *\right)\right)\right)-D_{\hat{\alpha}_{i}}^{k-1}\left(A\left(X\left(r_{i}, *\right)\right)\right)\right],\right. \\
& \left.e_{j_{i}}(*)\right\rangle_{\mathcal{H}} \\
& V_{2, \alpha}^{n}(t, x)=\sum_{i=1}^{k} \int_{0}^{t}\left\langle S(t-s, x-*) D_{\hat{\alpha}_{i}}^{k-1}\left(B\left(X_{n}(s, *)\right)\right), e_{j_{i}}(*)\right\rangle_{\mathcal{H}} \\
& \quad \times \sum_{\ell=1}^{2^{n}} 2^{n} T^{-1} 1_{\Delta_{\ell-1}}\left(r_{i}\right) 1_{\Delta_{\ell}}(s) d s-\left\langle S\left(t-r_{i}, x-*\right) D_{\hat{\alpha}_{i}}^{k-1}\left(B\left(X\left(r_{i}, *\right)\right)\right),\right. \\
& \left.e_{j_{i}}(*)\right\rangle_{\mathcal{H}}
\end{aligned}
$$




$$
\begin{aligned}
& V_{3, \alpha}^{n}(t, x)=\sum_{j \geq 0} \int_{\bigvee r_{i}}^{t}\left\langle S(t-s, x-*)\left[\Delta_{\alpha}\left(G, X_{n}(s, *)\right)-\Delta_{\alpha}(G, X(s, *))\right], e_{j}(*)\right\rangle_{\mathcal{H}} \\
& \times h_{j}(s) \mathrm{d} s, \\
& V_{4, \alpha}^{n}(t, x)=\int_{\bigvee_{r_{i}}}^{t} \int_{\mathbb{R}^{2}} S(t-s, x-y)\left(\Delta_{\alpha}\left(b, X_{n}(s, y)\right)-\Delta_{\alpha}(b, X(s, y))\right) \\
& \times \mathrm{d} s \mathrm{~d} y, \\
& V_{5, \alpha}^{n}(t, x)=\sum_{j \geq 0} \int_{\bigvee r_{i}}^{t}\left\langleS ( t - s , x - * ) \left[ G^{\prime}\left(X_{n}(s, *)\right) D_{\alpha}^{k} X_{n}(s, *)-G^{\prime}(X(s, x))\right.\right. \\
& \left.\left.\times D_{\alpha}^{k} X(s, *)\right], e_{j}(*)\right\rangle_{\mathcal{H}} h_{j}(s) \mathrm{d} s, \\
& V_{6, \alpha}^{n}(t, x)=\int_{\bigvee r_{i}}^{t} \int_{\mathbb{R}^{2}} S(t-s, x-y)\left[b^{\prime}\left(X_{n}(s, y)\right) D_{\alpha}^{k} X_{n}(s, y)-b^{\prime}(X(s, y)) D_{\alpha}^{k} X(s, y)\right] \\
& \times \mathrm{d} s \mathrm{~d} y, \\
& V_{7, \alpha}^{n}(t, x)=\sum_{j \geq 0} \int_{\bigvee r_{i}}^{t}\left\langle S(t-s, x-*) \Delta_{\alpha}\left(A, X_{n}(s, *)\right), e_{j}(*)\right\rangle_{\mathcal{H}} W_{j}(\mathrm{~d} s) \\
& +\sum_{j=0}^{n} \int_{\bigvee r_{i}}^{t}\left\langle S(t-s, x-*) \Delta_{\alpha}\left(B, X_{n}(s, *)\right), e_{j}(*)\right\rangle_{\mathcal{H}} \dot{W}_{j}^{n}(s) \mathrm{d} s \\
& -\sum_{j \geq 0}^{n} \int_{\bigvee r_{i}}^{t}\left\langle S(t-s, x-*) \Delta_{\alpha}(A+B, X(s, *)), e_{j}(*)\right\rangle_{\mathcal{H}} W_{j}(\mathrm{~d} s), \\
& V_{8, \alpha}^{n}(t, x)=\sum_{j \geq 0} \int_{\bigvee r_{i}}^{t}\left\langle S(t-s, x-*) A^{\prime}\left(X_{n}(s, *)\right) D_{\alpha}^{k} X_{n}(s, *), e_{j}(*)\right\rangle_{\mathcal{H}} W_{j}(\mathrm{~d} s) \\
& +\sum_{j=0}^{n} \int_{\bigvee r_{i}}^{t}\left\langle S(t-s, x-*) B^{\prime}\left(X_{n}(s, *)\right) D_{\alpha}^{k} X_{n}(s, *), e_{j}(*)\right\rangle_{\mathcal{H}} \dot{W}_{j}^{n}(s) \mathrm{d} s \\
& -\sum_{j \geq 0}^{n} \int_{\bigvee r_{i}}^{t}\left\langle S(t-s, x-*)(A+B)^{\prime}(X(s, *)) D_{\alpha}^{k} X(s, *), e_{j}(*)\right\rangle_{\mathcal{H}} W_{j}(\mathrm{~d} s) .
\end{aligned}
$$

Let $U_{i}^{n}(t, x)=E\left(\left\|V_{i}^{n}(t, x)\right\|_{H_{T}^{\otimes k}}^{p} 1_{A_{n}^{\gamma}(t)}\right), i=1, \ldots, 8$.

We have to prove that

$$
\lim _{n \rightarrow \infty} \sup _{(t, x) \in K_{m}^{T}} U_{\rho}^{n}(t, x)=0
$$

for any $\rho=1,2,3,4,7$, and

$$
U_{\rho}^{n}(t, x) \leq C \int_{\bigvee r_{i}(u, x) \in K_{m+T}^{s}}^{t} \sup _{\|} E\left(\left\|D^{k}\left(X_{n}(u, x)-X(u, x)\right)\right\|_{H_{T}^{\otimes k}}^{p} 1_{A_{n}^{\gamma}(u)}\right) \mathrm{d} s,
$$

for $\rho=5,6,8$, where $K_{m}^{t}=[0, t] \times\left\{x \in \mathbb{R}^{2}:\|x\| \leq m\right\}, t \in[0, T]$ and $m \in \mathbb{N}$, and we are assuming that (3.3) holds up to the order of derivation $k-1$. This can be done using the same ideas as in the proof of the above mentioned Proposition 2.3 in [11] taking into account the results given in the next Lemmas 1 to 3 . Basically, the proof of (3.9) for $\rho=1,2,3,4$ follows easily from the induction hypothesis, the proof for $\rho=2$ uses Lemma 3 and induction; the proof of (3.10) for $\rho=5,6$ uses the boundedness and Lipschitz properties of the coefficients. 
Notice that the structure of $U_{\rho}^{n}(t, x)$ for $\rho=7,8$ is similar. These terms are the most difficult to study. However the ideas used in Proposition 2.3 in [11] can be adapted using the additional ingredient of induction for the term corresponding to $\rho=7$. Once (3.9) and (3.10) have been established, the proof of the theorem is completed by means of a Gronwall-type argument.

The next statements correspond to the appropriate extensions of Lemmas 2.2-2.4 in [11], they are proved by induction on the derivative order $k$. The third one is related with the result stated in Proposition 2.2 in [11], we give a complete proof of it; as a by-product of this lemma we obtain Hölder continuity in time of the Malliavin derivative process $D^{k} X(t, x), t \geq 0$.

Lemma 1. We assume the hypothesis of Theorem 2. Then, for any $c \geq 0, k \in \mathbb{Z}_{+}, p \in[1, \infty)$, every integer $n \geq 1$ and $0<\beta<\beta_{0} \wedge 1$,

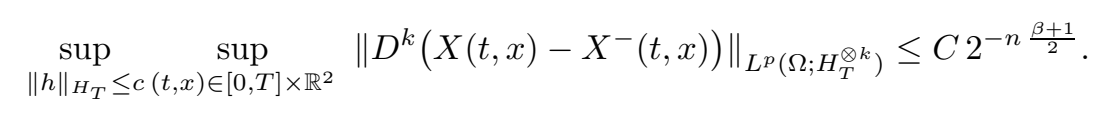

Lemma 2. We suppose that the assumptions of Theorem 2 are satisfied. For any $k \in \mathbb{Z}_{+}, p \in[1, \infty), \gamma>$ $2 T \ln 2, c \geq 0$, every integer $n \geq 1$ and $0<\beta<\beta_{0} \wedge 1$,

$$
\begin{aligned}
& \sup _{\|h\|_{H_{T} \leq c} \leq(t, x) \in[0, T] \times \mathbb{R}^{2}} \sup _{\leq}\left\|D^{k}\left(X_{n}(t, x)-X_{n}^{-}(t, x)\right) 1_{A_{n}^{\gamma}(t)}\right\|_{L^{p}\left(\Omega ; H_{T}^{\otimes k}\right)} \\
& \leq C n 2^{-n \frac{1+\beta}{2}}\left(1+\sup _{(t, x) \in[0, T] \times \mathbb{R}^{2}}\left\|D^{k}\left(X_{n}(t, x)\right) 1_{A_{n}^{\gamma}(t)}\right\|_{L^{p}\left(\Omega ; H_{T}^{\otimes k}\right)}\right), \\
& \sup _{\|h\|_{H_{T}} \leq c n \geq 1} \sup _{(t, x) \in[0, T] \times \mathbb{R}^{2}}\left\|D^{k}\left(X_{n}(t, x)+X_{n}^{-}(t, x)\right) 1_{A_{n}^{\gamma}(t)}\right\|_{L^{p}\left(\Omega ; H_{T}^{\otimes k}\right)}<\infty .
\end{aligned}
$$

Consequently,

$$
\sup _{\|h\|_{H_{T}} \leq c(t, x) \in[0, T] \times \mathbb{R}^{2}} \sup \left\|D^{k}\left(X_{n}(t, x)-X_{n}^{-}(t, x)\right) 1_{A_{n}^{\gamma}(t)}\right\|_{L^{p}\left(\Omega ; H_{T}^{\otimes k}\right)} \leq C n 2^{-n \frac{1+\beta}{2}} .
$$

Lemma 3. We assume the hypothesis of Theorem 2. Then, for any $c \geq 0, p \in[1, \infty), 0 \leq t \leq t^{\prime} \leq T$, $k \in \mathbb{Z}_{+}, \alpha \in\left(0, \frac{1}{2}\left(\beta_{0} \wedge 1\right)\right)$, we have

$$
\sup _{\|h\|_{H_{T}} \leq c}\left\|D^{k}\left(X(t, x)-X\left(t^{\prime}, x\right)\right)\right\|_{L^{p}\left(\Omega ; H_{T}^{\otimes k}\right)} \leq C\left|t-t^{\prime}\right|^{\alpha} .
$$

Proof. Fix $0 \leq t \leq t^{\prime} \leq T, x \in \mathbb{R}^{2}$; set $\gamma\left(t, t^{\prime}, x ; s, y\right)=S(t-s, x-y)-S\left(t^{\prime}-s, x-y\right),(s, y) \in[0, T] \times \mathbb{R}^{2}$. Notice that $(s, y, z) \longmapsto \gamma\left(t, t^{\prime}, x ; s, y\right) f(|y-z|) \gamma\left(t, t^{\prime}, x ; s, z\right)$ defines a density on $[0, T] \times \mathbb{R}^{2} \times \mathbb{R}^{2}$.

We first prove (3.12) for $k=0$. For any fixed $p \in[1, \infty)$ we set

$$
E\left(\left|X(t, x)-X\left(t^{\prime}, x\right)\right|^{p}\right) \leq C \sum_{i=1}^{4} R_{i}\left(t, t^{\prime} ; x\right),
$$


with

$$
\begin{aligned}
& R_{1}\left(t, t^{\prime} ; x\right)=\left|X^{0}(t, x)-X^{0}\left(t^{\prime}, x\right)\right|^{p}, \\
& R_{2}\left(t, t^{\prime} ; x\right)=E\left(\left|\sum_{j \geq 0} \int_{0}^{T}\left\langle\gamma\left(t, t^{\prime}, x ; s, *\right)(A+B)(X(s, *)), e_{j}(*)\right\rangle_{\mathcal{H}} W_{j}(\mathrm{~d} s)\right|^{p}\right), \\
& R_{3}\left(t, t^{\prime} ; x\right)=E\left(\left|\sum_{j \geq 0} \int_{0}^{T}\left\langle\gamma\left(t, t^{\prime}, x ; s, *\right) G(X(s, *)), e_{j}(*)\right\rangle_{\mathcal{H}} h_{j}(s) \mathrm{d} s\right|^{p}\right), \\
& R_{4}\left(t, t^{\prime} ; x\right)=E\left(\mid \int_{0}^{T} \gamma\left(t, t^{\prime}, x ; s, y\right) b\left(\left.X(s, y) \mathrm{d} s \mathrm{~d} y\right|^{p}\right) .\right.
\end{aligned}
$$

In the proof of Proposition 1.4 in [10] we have established that

$$
R_{1}\left(t, t^{\prime} ; x\right) \leq C\left\{\left\|v_{0}\right\|_{q_{0}}^{p}\left|t-t^{\prime}\right|^{p\left(\frac{1}{q}-\frac{1}{2}\right)}+\left|t-t^{\prime}\right|^{\frac{p}{2}\left(\beta_{0} \wedge 1\right)}\right\}
$$

where $\frac{1}{q}+\frac{1}{q_{0}}=1$ and we have used (C2). The restriction on $q_{0}$ yields, for $\alpha \in\left(0, \frac{1}{2}\left(\beta_{0} \wedge 1\right)\right)$,

$$
R_{1}\left(t, t^{\prime} ; x\right) \leq C\left|t-t^{\prime}\right|^{\alpha p}
$$

Burkholder's inequality, then Fubini's theorem and finally Hölder's inequality with respect to the measure whose density has been described at the begining of the proof, yield

$$
R_{2}\left(t, t^{\prime} ; x\right) \leq C\left\|\gamma\left(t-t^{\prime}, x ; \cdot, *\right)\right\|_{L^{2}([0, T] ; \mathcal{H})}^{p} \sup _{(s, x) \in[0, T] \times \mathbb{R}^{2}} E\left(|(A+B)(X(s, x))|^{p}\right)
$$

In [11], Lemma 2.5 (see the proof of $(2.42)$ with $x=\bar{x}$ ), it is proved that

$$
\left\|\gamma\left(t, t^{\prime}, x, \cdot, *\right)\right\|_{L^{2}([0, T] ; \mathcal{H})}^{2} \leq \mu_{t, t^{\prime}-t}+\tilde{\mu}_{t, t^{\prime}-t}+2\left(\mu_{t, t^{\prime}-t} \tilde{\mu}_{t, t^{\prime}-t}\right)^{1 / 2}
$$

with $\mu_{t, h}, \tilde{\mu}_{t, h}$ are defined in (A.2, A.3), respectively. The bound (A.5) yields

$$
R_{2}\left(t, t^{\prime} ; x\right) \leq C\left(\left|t-t^{\prime}\right|^{\alpha p}\right)
$$

with $\alpha \in\left(0, \frac{1}{2}\left(\beta_{0} \wedge 1\right)\right)$.

The same bound is obtained for the remaining terms $R_{i}\left(t, t^{\prime} ; x\right), i=3,4$, following similar ideas.

The proof for any integer $k \geq 1$ is completed using induction on $k$.

We continue by setting the additional ingredients needed in the application of Proposition 1 in our example.

Lemma 4. Let $\underline{z}=\left(z_{1}, \ldots, z_{d}\right)$. For any integer $k \geq 0$ and any $h \in H_{T}$ set

$$
\rho_{n}^{k, h, \underline{z}}(t, \underline{x})(\omega)=D^{k} u(t, \underline{x})\left(W-W^{n}+\int_{0}\left(h+\sum_{i=1}^{d} z_{i} \bar{D} \Phi^{h}\left(t, x_{i}\right)\right)(s) \mathrm{d} s\right),
$$

where $\bar{D}$ denotes the Fréchet derivative operator. Then, for any $p \geq 1$,

$$
\lim _{M \rightarrow \infty} \sup _{n} P\left\{\int_{|\underline{z}| \leq 1}\left\|\rho_{n}^{k, h, \underline{z}}(t, \underline{x})\right\|_{H_{T}^{\otimes k}}^{p} \mathrm{~d} z>M\right\}=0 .
$$


Proof. Fix $\varepsilon>0$. By property (2.17) there exists $n_{0} \in \mathbb{N}$ such that for any $n \geq n_{0}$ and $\gamma>2 T \ell n 2, P\left(A_{n}^{\gamma}(t)^{c}\right)$ $<\varepsilon$. Then, Chebychev's inequality yields, for any $n \geq n_{0}$,

$$
P\left\{\int_{|\underline{z}| \leq 1}\left\|\rho_{n}^{k, h, \underline{z}}(t, \underline{x})\right\|_{H \otimes k}^{p} \mathrm{~d} z>M\right\} \leq \varepsilon+\frac{1}{M} \sup _{|\underline{z}| \leq 1} E\left(\left\|\rho_{n}^{k, h, \underline{z}}(t, \underline{x})\right\|_{H_{T}^{\otimes k}}^{p} 1_{A_{n}^{\gamma}(t)}\right) .
$$

Thus, it suffices to check that

$$
\sup _{n} \sup _{|\underline{z}| \leq 1} E\left(\left\|\rho_{n}^{k, h, \underline{z}}(t, \underline{x})\right\|_{H_{T}^{\otimes k}}^{p} 1_{A_{n}^{\gamma}(t)}\right) \leq C<\infty .
$$

The equation satisfied by $D^{k} u(t, x),(t, x) \in[0, T] \times \mathbb{R}^{2}$, is obtained from equation (3.8) with $A=G=0, B=\sigma$. Set $\tilde{W}^{n, h, \underline{z}}=W-W^{n}+\int_{0}\left(h+\sum_{i=1}^{d} z_{i} \bar{D} \Phi^{h}\left(t, x_{i}\right)\right)(s) \mathrm{d} s$; then, each component of the $d$-dimensional random vector $\rho_{n}^{k, h, \underline{z}}(t, \underline{x})$ satisfies the following equation, where for simplicity we have omitted the index of the variable $x$ :

$$
\begin{aligned}
\rho_{n, \alpha}^{k, h, \underline{z}}(t, x)= & \sum_{i=1}^{k}\left\langle D_{\hat{\alpha}_{i}}^{k-1}\left(\sigma\left(u\left(r_{i}, *\right)\right)\right)\left(\tilde{W}^{n, h, \underline{z}}\right) S\left(t-r_{i}, x-*\right), e_{j_{i}}(*)\right\rangle_{\mathcal{H}} \\
& +\sum_{j \geq 0} \int_{\bigvee_{i} r_{i}}^{t}\left\langle S(t-s, x-*) \Delta_{\alpha}(\sigma, u(s, *))\left(\tilde{W}^{n, h, \underline{z}}\right), e_{j}(*)\right\rangle_{\mathcal{H}} W_{j}(\mathrm{~d} s) \\
& +\sum_{j \geq 0} \int_{\bigvee_{i} r_{i}}^{t}\left\langle S(t-s, x-*) \Delta_{\alpha}(\sigma, u(s, *))\left(\tilde{W}^{n, h, \underline{z}}\right), e_{j}(*)\right\rangle_{\mathcal{H}}\left(h_{j}(s)\right. \\
& \left.-\dot{W}_{j}^{n}(s)+\sum_{i=1}^{d} z_{i} \bar{D}_{s, j} \Phi^{h}\left(t, x_{i}\right)\right) \mathrm{d} s \\
& +\int_{\bigvee_{i} r_{i}}^{t} \int_{\mathbb{R}^{2}} S(t-s, x-y) \Delta_{\alpha}(b, u(s, y))\left(\tilde{W}^{n, h, \underline{z}}\right) \mathrm{d} s \mathrm{~d} y \\
& +\sum_{j \geq 0} \int_{\bigvee_{i} r_{i}}^{t}\left\langle S(t-s, x-*) \sigma^{\prime}\left(\rho_{n}^{0, h, \underline{z}}(s, *)\right) \rho_{n, \alpha}^{k, h, \underline{z}}(s, *), e_{j}(*)\right\rangle_{\mathcal{H}} W_{j}(\mathrm{~d} s) \\
& +\sum_{j \geq 0} \int_{\bigvee_{i} r_{i}}^{t}\left\langle S(t-s, x-*) \sigma^{\prime}\left(\rho_{n}^{0, h, \underline{z}}(s, *)\right) \rho_{n, \alpha}^{k, h, \underline{z}}(s, *), e_{j}(*)\right\rangle_{\mathcal{H}}\left(h_{j}(s)\right. \\
& \left.-\dot{W}_{j}^{n}(s)+\sum_{i=1}^{d} z_{i} \bar{D}_{s, j} \Phi^{h}\left(t, x_{i}\right)\right) \mathrm{d} s \\
& +\int_{\bigvee_{i} r_{i}}^{t} \int_{\mathbb{R}^{2}} S(t-s, x-y) b^{\prime}\left(\rho_{n}^{0, h, z}(s, y)\right) \rho_{n, \alpha}^{k, h, \underline{z}}(s, y) \mathrm{d} s \mathrm{~d} y .
\end{aligned}
$$

Notice that, by the chain rule $(2.20)$

$$
D_{\hat{\alpha}_{i}}^{k-1}(\sigma(u(s, x)))\left(\tilde{W}^{n, h, \underline{z}}\right)=\sum_{m=1}^{k-1} \sum_{P_{m}} c_{m} \sigma^{(m)}\left(\rho_{n}^{0, h, \underline{z}}(s, x)\right) \prod_{j=1}^{m} \rho_{n, p_{i}}^{\left|p_{i}\right|, h, \underline{z}}(s, x),
$$

where if $\alpha=\left(\alpha_{1}, \ldots, \alpha_{k}\right), P_{m}$ is the set of partitions of the set $\left\{\left(\alpha_{1}, \ldots, \alpha_{i-1}, \alpha_{i+1} \ldots \alpha_{k}\right) \in\left(\mathbb{R}_{+} \times \mathbb{N}\right)^{k-1}\right\}$ consisting of $m$ disjoint subsets $p_{1}, \ldots, p_{m}, m=1, \ldots, k-1$. Moreover, for $g=\sigma, b$,

$$
\Delta_{\alpha}(g, u(s, x))\left(\tilde{W}^{n, h, \underline{z}}\right)=D_{\alpha}^{k}(g(u(s, x)))\left(\tilde{W}^{n, h, \underline{z}}\right)-g^{\prime}\left(\rho_{n}^{0, h, \underline{z}}(s, x)\right) \rho_{n, \alpha}^{k, h} \underline{\underline{z}}(s, x) .
$$


Therefore equation (3.16) has the same structure than equation (3.7) with $h_{j}$ replaced by $h_{j}+\sum_{i=1}^{d} z_{i} \bar{D} .{ }^{\prime} j$ $\Phi^{h}\left(t, x_{i}\right)$. We point out in the Appendix that, for any $a \geq 0,(t, x) \in[0, T] \times \mathbb{R}^{2}, \sup _{\|h\|_{H_{T}} \leq a}\left\|\bar{D} \Phi^{h}(t, x)\right\|_{H_{T}}$ $\leq C<\infty$. Thus, Lemma 2 (see (3.11)) shows (3.15) and completes this proof.

Remark 1. Consider the transformation defined in (2.15), $T_{n}^{h}(\omega)=\left(W_{j}-W_{j}^{n}+\int_{0} h_{j}(s) \mathrm{d} s\right)_{j \geq 0}, h \in H_{T}$. Using the notation introduced in Lemma \& we have that $u(t, x)\left(T_{n}^{h}\right)=\rho_{n}^{0, h, \underline{\underline{0}}}(t, x)$ and $D u(t, x)\left(T_{n}^{h}\right)=\rho_{n}^{1, h, \underline{0}}(t, x)$. Hence (3.16) yields

$$
\begin{aligned}
\rho_{n, \alpha}^{1, h, \underline{0}}(t, x)= & \left\langle S(t-r, x-*) \sigma\left(\rho_{n}^{0, h, \underline{0}}(r, *)\right), e_{j}(*)\right\rangle_{\mathcal{H}} \\
& +\sum_{k \geq 0} \int_{r}^{t}\left\langle S(t-s, x-*) \sigma^{\prime}\left(\rho_{n}^{0, h, \underline{0}}(r, *)\right), \rho_{n, \alpha}^{1, h, 0}(s, *) e_{k}(*)\right\rangle_{\mathcal{H}} \\
& \times\left\{W_{k}(\mathrm{~d} s)-\dot{W}_{k}^{n}(s) \mathrm{d} s+h_{k}(s) \mathrm{d} s\right\} \\
& +\int_{r}^{t} \int_{\mathbb{R}^{2}} S(t-s, x-y) b^{\prime}\left(\rho_{n}^{0, h, 0}(s, y)\right) \rho_{n, \alpha}^{1, h, 0}(s, y) \mathrm{d} s \mathrm{~d} y,
\end{aligned}
$$

with $\alpha=(r, j) \in[0, T] \times \mathbb{N}$.

Then, Theorem 2 with $k=1$ and the coefficients $A=-B=G=\sigma$ yields

$$
\lim _{n \rightarrow \infty} \sup _{0 \leq t \leq T} \sup _{\underline{x} \in K^{d}} E\left(\left\|(D u(t, \underline{x})) \circ T_{n}^{h}-\bar{D} \Phi^{h}(t, \underline{x})\right\|_{H_{T}\left(\mathbb{R}^{d}\right)}^{p} 1_{A_{n}^{\gamma}(t)}\right)=0 .
$$

We conclude this section by completing Step 1 of our programme, as has been described in Section 1. Recall that $p_{t, \underline{x}}(y)$ denotes the density of the random vector $\left(u\left(t, x_{1}\right), \ldots, u\left(t, x_{d}\right)\right)$ at $y \in \mathbb{R}^{d}$.

Theorem 3. We assume

(i') there exist $0<a_{2} \leq a_{1}<2$ such that $2\left(a_{1}-a_{2}\right)<a_{2} \wedge 1$, and a positive constant $C_{1}$ such that for $t \in[0, T], C_{1} t^{a_{1}} \leq \int_{0}^{t} y f(y) \ln \left(1+\frac{t}{y}\right) \mathrm{d} y$ and $\int_{0^{+}} y^{1-a_{2}} f(y) \mathrm{d} y<\infty ;$

(ii') $u_{0}: \mathbb{R}^{2} \rightarrow \mathbb{R}$ is of class $\mathcal{C}^{1}$, bounded, with $\frac{1}{2}\left(a_{2} \wedge 1\right)$-Hölder continuous partial derivatives, $\nabla u_{0} \in L^{q_{1}}\left(\mathbb{R}^{2}\right)$ for some $q_{1}>2 ; v_{0}: \mathbb{R}^{2} \rightarrow \mathbb{R}$ belongs to $L^{q_{0}}\left(\mathbb{R}^{2}\right)$ for some $\left.\left.q_{0} \in\right] 4 \vee \frac{2}{1-\left(a_{2} \wedge 1\right)}, \infty\right] ;$

(iii) the coefficients $\sigma$ and $b$ are $\mathcal{C}^{\infty}$ functions with bounded derivatives of any order $i \geq 1$;

(iv) there exists $a>0$ such that $\left|\sigma\left(u\left(t, x_{j}\right)\right)\right| \geq a$, for any $j=1, \ldots, d$, a.s.

Then the next two statements on $y \in \mathbb{R}^{d}$ are equivalent: (a) $p_{t, \underline{x}}(y)>0$ and (b) there exists $h \in H_{T}$ such that $\Phi^{h}(t, \underline{x})=y$ and $\operatorname{det} \gamma_{\Phi^{h}(t, \underline{x})}>0$.

Proof. First we establish $(b) \Rightarrow(a)$. With this purpose, we apply Proposition 1 to $F=\left(u\left(t, x_{1}\right), \ldots, u\left(t, x_{d}\right)\right)$. Let $v_{n}=u \circ T_{n}^{h}$ with $T_{n}^{h}$ defined by (2.15). The process $\left\{v_{n}(t, x),(t, x) \in[0, T] \times \mathbb{R}^{2}\right\}$ satisfies the equation

$$
\begin{aligned}
v_{n}(t, x)= & X^{0}(t, x)+\sum_{j \geq 0} \int_{0}^{t}\left\langle S(t-s, x-*) \sigma\left(v_{n}(s, *)\right), e_{j}(*)\right\rangle_{\mathcal{H}}\left\{W_{j}(\mathrm{~d} s)\right. \\
& \left.-\dot{W}_{j}^{n}(s) d s+h_{j}(s) d s\right\}+\int_{0}^{t} \int_{\mathbb{R}^{2}} S(t-s, x-y) b\left(v_{n}(s, y)\right) \mathrm{d} s \mathrm{~d} y,
\end{aligned}
$$


which is a particular case of equation (3.1) with $A=-B=G=\sigma$. For this choice of coefficients, equation (3.2) coincides with that satisfied by the skeleton $\Phi^{h}(t, x)$ (see (1.9)). Then, Theorem 2 yields

$$
\lim _{n \rightarrow \infty} \sup _{0 \leq t \leq T} \sup _{\underline{x} \in K^{d}} E\left(\left\|\left(u \circ T_{n}^{h}\right)(t, \underline{x})-\Phi^{h}(t, x)\right\|^{p} 1_{A_{n}^{\gamma}(t)}\right)=0,
$$

for any $p \in(1, \infty), \gamma>2 T \ell n 2$ and every compact set $K \subset \mathbb{R}^{2}$. By (2.17) this ensures condition (2.2).

The validity of (2.3) follows from (3.17) and (2.17). Finally (2.4) has been proved in Lemma 4.

Let us now check $(a) \Rightarrow(b)$. Consider the process defined by the evolution equation

$$
\begin{aligned}
u_{n}(t, x)= & X^{0}(t, x)+\sum_{j \geq 0} \int_{0}^{t}\left\langle S(t-s, x-*) \sigma\left(u_{n}(s, *)\right), e_{j}(*)\right\rangle_{\mathcal{H}} \dot{W}_{j}^{n}(s) \mathrm{d} s \\
& +\int_{0}^{t} \int_{\mathbb{R}^{2}} S(t-s, x-y) b\left(u_{n}(s, y)\right) \mathrm{d} s \mathrm{~d} y .
\end{aligned}
$$

This is a particular case of equation (3.1) with $A=G=0, B=\sigma$. Moreover, the process $\left\{u_{n}(t, x),(t, x)\right.$ $\left.\in[0, T] \times \mathbb{R}^{2}\right\}$ coincides with the skeleton $\left\{\Phi^{\dot{W}^{n}}(t, x),(t, x) \in[0, T] \times \mathbb{R}^{2}\right\}$; with this choice of coefficients, equation (3.2) coincides with (1.3). Theorem 2 shows that the assumptions of Proposition 2 are satisfied with $\Phi(h)=\Phi^{h}(t, \underline{x}), F=u(t, \underline{x})$ and the localizing sequence $\left(A_{n}^{\gamma}(t)\right)_{n \in \mathbb{N}}$ defined in (2.16). This completes de proof of the theorem.

\section{Positivity of the Density}

The purpose of this section is to analize under which conditions property (b) of Theorem 3 is satisfied for any $y \in \mathbb{R}^{d}$, that is, the density $p_{t, \underline{x}}(y)$ is strictly positive everywhere.

Theorem 4. Suppose that the assumptions ( $i$, ii', iii) of Theorem 3 are satisfied. Moreover, we assume

(iv') $\{\inf |\sigma(z)|, z \in \mathbb{R}\}>C$, for some constant $C>0$;

(v) the covariance functional $J$ defined in (1.8) is positive.

Then, for any $y \in \mathbb{R}^{d}$ there exists $h \in H_{T}$ such that $\Phi^{h}(t, \underline{x})=y$ and $\operatorname{det} \gamma_{\Phi^{h}(t, \underline{x})}>0$.

Proof. The nondegeneracy of the matrix $\gamma_{\Phi^{h}(t, \underline{x})}$ has been established in Proposition 3 of the Appendix. Hence it only remains to prove that each $y \in \mathbb{R}^{d}$ can be reached, with an appropriate choice of $h \in H_{T}$, through the skeleton.

Set $y=\left(y_{1}, \ldots, y_{d}\right)$ and let $\lambda_{1}, \ldots, \lambda_{d} \in \mathbb{R}$ satisfying the linear system

$$
y_{\ell}-X^{0}\left(t, x_{\ell}\right)=\sum_{i=1}^{d} \lambda_{i} \gamma_{S}^{i, \ell}, \quad \ell=1, \ldots, d
$$

where $\gamma_{S}^{i, \ell}=\left\langle S\left(t-\cdot, x_{i}-*\right), S\left(t-\cdot, x_{\ell}-*\right)\right\rangle_{L^{2}([0, T] ; \mathcal{H})}$.

Let $k(s, z)=\sum_{i=1}^{d} \lambda_{i} S\left(t-s, x_{i}-z\right), K(s, y)=\int_{\mathbb{R}^{2}} f(|y-z|) k(s, z) \mathrm{d} z, s \in[0, T], z, y \in \mathbb{R}^{2}$. Notice that $k \in L^{2}([0, T] ; \mathcal{H})$ and by Schwarz's inequality

$$
\int_{0}^{T} \mathrm{~d} s \int_{\mathbb{R}^{2}} \mathrm{~d} y|K(s, y)| S(t-s, x-y)<\infty
$$

For any $\tau \in[0, T], x \in \mathbb{R}^{2}$, set

$$
\varphi(\tau, x)=X^{0}(\tau, x)+\int_{0}^{\tau} \mathrm{d} s \int_{\mathbb{R}^{2}} \mathrm{~d} y K(s, y) S(\tau-s, x-y) .
$$


By construction, $\varphi\left(t, x_{\ell}\right)=y_{\ell}, \ell=1, \ldots, d$.

Let $\mathcal{Y}$ be the linear space consisting of functions $H:[0, T] \times \mathbb{R}^{2} \longrightarrow \mathbb{R}$ such that $\int_{0}^{T} \mathrm{~d} s \int_{\mathbb{R}^{2}} \mathrm{~d} y|H(s, y)| S(t-$ $s, x-y)<\infty$. Consider the linear operator

$$
\begin{aligned}
\mathcal{T}=L^{2}([0, T] ; \mathcal{H}) & \longrightarrow \mathcal{Y} \\
h & \longmapsto \int_{\mathbb{R}^{2}} f(|y-z|) h(s, z) \mathrm{d} z
\end{aligned}
$$

Hypothesis (v) ensures that $\mathcal{T}$ admits an inverse operator $\mathcal{T}^{-1}$. Indeed, 0 is not an eigenvalue of $\mathcal{T}$.

Set

$$
H_{0}(s, y)=-[b(\varphi(s, y))-K(s, y)] \sigma(\varphi(s, y))^{-1},(s, y) \in[0, T] \times \mathbb{R}^{2}
$$

It is not difficult to check that $H_{0} \in \mathcal{Y}$. Therefore

$$
H_{0}(s, y)=\int_{\mathbb{R}^{2}} f(|y-z|) h_{0}(s, z) \mathrm{d} z,
$$

for some $h_{0} \in L^{2}([0, T] ; \mathcal{H})$. Let $h_{0}^{j}(s)=\left\langle h_{0}(s, *), e_{j}(*)\right\rangle_{\mathcal{H}}, j \geq 0$. Then $\left(h_{0}^{j}, j \geq 0\right) \in H_{T}$.

Substituting the function $K=H_{0}$ given by (4.3) in (4.2) we conclude that $\Phi^{h_{0}} \equiv \varphi$, by uniqueness of solution, and consequently $\Phi^{h_{0}}\left(t, x_{\ell}\right)=y_{\ell}, \ell=1, \ldots, d$, as we wanted to prove.

\section{Appendix}

Let $S(t, x),(t, x) \in\left[0, \infty\left[\times R^{2}\right.\right.$ be the fundamental solution of the stochastic wave equation. We start this section by quoting some notations and results concerning $S$ that have been proved in [10] and [8] and used along the proofs.

For any $t \in[0, T], h \geq 0$, set

$$
\begin{gathered}
J(t)=\int_{|y|<|x|<t} \frac{1}{\sqrt{t^{2}-|x|^{2}}} f(|x-y|) \frac{1}{\sqrt{t^{2}-|y|^{2}}} \mathrm{~d} x \mathrm{~d} y, \\
\mu(t)=\int_{0}^{t} \mathrm{~d} s \int_{\mathbb{R}^{2}} \mathrm{~d} x \int_{\mathbb{R}^{2}} \mathrm{~d} y S(s, x) f(|x-y|) S(s, y)=\frac{1}{2 \pi^{2}} \int_{0}^{t} J(s) \mathrm{d} s, \\
\mu_{t, h}=\int_{0}^{t} \mathrm{~d} s \int_{|y|<s} \mathrm{~d} y \int_{|z|<s} \mathrm{~d} z[S(s, y)-S(s+h, y)] f(|y-z|) \\
\times[S(s, z)-S(s+h, z)], \\
\tilde{\mu}_{t, h}=\int_{0}^{t} \mathrm{~d} s \int_{s \leq|y|<s+h}^{t} \mathrm{~d} s \int_{|x|<s} \frac{\mathrm{d} x}{\sqrt{s^{2}-|x|^{2}}}=\frac{t^{2}}{2},
\end{gathered}
$$


Assume that $f$ satisfies the assumption (C1) of Section 3; then Lemma A.1 in [10] implies

$$
J(t) \leq C t^{\beta}, \mu(t) \leq C t^{\beta+1}, t \in[0, T], \beta<\beta_{0} .
$$

Moreover, for $h$ small enough and $0<\delta<\beta_{0} \wedge 1$,

$$
\mu_{t, h}+\tilde{\mu}_{t, h} \leq C h^{\delta}
$$

(see Lem. A.5 in [10] and [8], Rem. A.6).

For fixed distinct points $x_{1}, \ldots, x_{d} \in \mathbb{R}^{2}$, set

$$
\gamma_{S}=\left(\left\langle S\left(t-\cdot, x_{i}-*\right), S\left(t-\cdot, x_{j}-*\right)\right\rangle_{L^{2}([0, T] ; \mathcal{H})}\right)_{1 \leq i, j \leq d}
$$

The next lemma provides one of the ingredients in the proofs of Section 4.

Lemma 5. Assume that there exist constants $C_{1}>0$ and $a_{1} \in(0,2)$ such that for $t \in[0, T]$,

$$
C_{1} t^{a_{1}} \leq \int_{0}^{t} y f(y) \ln \left(1+\frac{t}{y}\right) \mathrm{d} y .
$$

Then, $\operatorname{det} \gamma_{S}>0$.

Proof. It suffices to prove that for any $\underline{v}=\left(v_{1}, \ldots, v_{d}\right) \in \mathbb{R}^{d},|v|=1$,

$$
\Gamma(s, v):=v^{t} \gamma_{S} v=\int_{0}^{t} \mathrm{~d} r\left\|\sum_{i=1}^{d} v_{i} S\left(t-r, x_{i}-*\right)\right\|_{\mathcal{H}}^{2}>0 .
$$

Let $\varepsilon \in(0, t)$ to be determined later. Then $\Gamma(s, v) \geq I_{1}-\left|I_{2}\right|$ with

$$
\begin{aligned}
I_{1} & =\sum_{i=1}^{d} \int_{t-\varepsilon}^{t} v_{i}^{2}\left\|S\left(t-r, x_{i}-*\right)\right\|_{\mathcal{H}}^{2} \mathrm{~d} r=\mu(\varepsilon), \\
I_{2} & =\sum_{\substack{i, j=1 \\
i \neq j}}^{d} \int_{t-\varepsilon}^{t} v_{i} v_{j}\left\langle S\left(t-r, x_{i}-*\right), S\left(t-r, x_{j}-*\right)\right\rangle_{\mathcal{H}} \mathrm{d} r .
\end{aligned}
$$

Lemma A.1 [10] together with the assumption of the lemma yield $I_{1} \geq C \varepsilon^{a_{1}+1}$.

Let $m=\inf \left\{\left|x_{i}-x_{j}\right|, i \neq j\right\}, M=\sup \left\{\left|x_{i}-x_{j}\right|, i \neq j\right\}$. Then, if $4 \varepsilon<m$, for any $y \in \mathbb{R}^{2},\left|y-x_{i}\right| \leq \varepsilon, z \in$ $\mathbb{R}^{2},\left|z-x_{j}\right| \leq \varepsilon$, we have $\frac{m}{2} \leq|y-z| \leq 2 \varepsilon+M$. Therefore $\sup \left\{f(|y-z|),\left|y-x_{i}\right| \leq \varepsilon,\left|z-x_{j}\right| \leq \varepsilon\right\} \leq C$. Then

$$
\begin{aligned}
& \int_{t-\varepsilon}^{t}\left\langle S\left(t-r, x_{i}-*\right), S\left(t-r, x_{j}-*\right)\right\rangle_{\mathcal{H}} \mathrm{d} r \\
= & \int_{t-\varepsilon}^{t} \mathrm{~d} r \int_{\left|x_{i}-y\right| \leq t-r} \mathrm{~d} y \int_{\left|x_{j}-z\right| \leq t-r} \mathrm{~d} t S\left(t-r, x_{i}-y\right) f(|y-z|) S\left(t-r, x_{j}-z\right) \\
\leq & C \int_{t-\varepsilon}^{t} \mathrm{~d} r\left(\int_{|x-y| \leq t-r} S(t-r, x-y) \mathrm{d} y\right)^{2} \leq C \varepsilon^{3} .
\end{aligned}
$$

Since $a_{1}+1<3$, taking $\varepsilon$ small enough we obtain $I_{1}-\left|I_{2}\right|>0$. 
Fix $(t, x) \in[0, T] \times \mathbb{R}^{2}$ and assume (1.5) and the hypotheses (ii', iii) of Theorem 3. Then the map $h \in H_{T} \rightarrow$ $\Phi^{h}(t, x)$ is infinitely Fréchet differentiable. For any integer $k \geq 1$, the $k$-th order Fréchet derivative $\bar{D}^{k} \Phi^{h}(t, x)$ satifies a differentiable equation like (3.8) with $A=-B=G=\sigma$. In particular, for $k=1$.

$$
\begin{aligned}
\bar{D}_{r, j} \Phi^{h}(t, x)= & \left\langle S(t-r, x-*) \sigma\left(\Phi^{h}(r, *)\right), e_{j}(*)\right\rangle_{\mathcal{H}} \\
& +\sum_{k \geq 0} \int_{0}^{t} \mathrm{~d} s\left\langle S(t-s, x-*) \sigma^{\prime}\left(\Phi^{h}(s, *)\right) D_{r, j} \Phi^{h}(s, *), e_{k}(*)\right\rangle_{\mathcal{H}} h_{k}(s) \\
& +\int_{0}^{t} \int_{\mathbb{R}^{2}} S(t-s, x-y) b^{\prime}\left(\Phi^{h}(s, y)\right) D_{r, j} \Phi^{h}(s, y) \mathrm{d} s \mathrm{~d} y
\end{aligned}
$$

if $r \leq t$ and $\bar{D}_{r, j} \Phi^{h}(t, x)=0$, if $t<r$.

It is easy to check that for any $(t, x) \in[0, T] \times \mathbb{R}^{2}$,

$$
\sup _{\|h\|_{H_{T}} \leq a}\left\|\bar{D} \Phi^{h}(t, x)\right\|_{H_{T}} \leq C<\infty .
$$

We now prove the nondegeneracy of the deterministic Malliavin matrix

$$
\gamma_{\Phi^{h}(t, \underline{x})}=\left(\left\langle\bar{D} \Phi^{h}\left(t, x_{i}\right), \bar{D} \Phi^{h}\left(t, x_{j}\right)\right\rangle_{H_{T}}\right)_{1 \leq i, j \leq d} .
$$

Proposition 3. Assume $\left(i\right.$, ii', iii) and (iv'). Then $\operatorname{det} \gamma_{\Phi^{h}(t, \underline{x})}$ is strictly positive.

Proof. Set $\bar{D}_{r, j} \Phi^{h}(t, x)=\varphi_{r, j}(t, x)+\psi_{r, j}(t, x)$, with $\varphi_{r, j}(t, x)=\left\langle S(t-r, x-*) \sigma\left(\Phi^{h}(r, *)\right), e_{j}(*)\right\rangle_{\mathcal{H}}$. Let $v \in \mathbb{R}^{d},|v|=1$.

The triangle inequality yields

$$
v^{*} \gamma_{\Phi^{h}(t, \underline{x})} v=\sum_{k=0}^{\infty} \int_{0}^{t} \mathrm{~d} r\left|\sum_{i=1}^{d} v_{i} \bar{D}_{r, k} \Phi^{h}\left(t, x_{i}\right)\right|^{2} \geq \frac{1}{2} J_{1}-J_{2},
$$

with

$$
\begin{aligned}
& J_{1}=\sum_{k=0}^{\infty} \int_{t-\gamma}^{t} \mathrm{~d} r\left|\sum_{i=1}^{d} v_{i} \varphi_{r, k}\left(t, x_{i}\right)\right|^{2}, \\
& J_{2}=\sum_{k=0}^{\infty} \int_{t-\gamma}^{t} \mathrm{~d} r\left|\sum_{i=1}^{d} v_{i} \psi_{r, k}\left(t, x_{i}\right)\right|^{2},
\end{aligned}
$$

$\gamma \in(0, t)$ to be determined later.

Lower bound for $J_{1}$ : We write $J_{1} \geq \frac{1}{2} J_{11}-J_{12}$, where

$$
\begin{aligned}
J_{11} & =\sum_{k=0}^{\infty} \int_{t-\gamma}^{t} \mathrm{~d} r\left|\sum_{i=1}^{d} v_{i} \sigma\left(\Phi^{h}\left(r, x_{i}\right)\right)\left\langle S\left(t-r, x_{i}-*\right), e_{k}(*)\right\rangle_{\mathcal{H}}\right|^{2}, \\
J_{12} & =\sum_{k=0}^{\infty} \int_{t-\gamma}^{t} \mathrm{~d} r\left|\sum_{i=1}^{d} v_{i}\left\langle S\left(t-r, x_{i}-*\right)\left[\sigma\left(\Phi^{h}(r, *)\right)-\sigma\left(\Phi^{h}\left(r, x_{i}\right)\right)\right], e_{k}(*)\right\rangle_{\mathcal{H}}\right|^{2} .
\end{aligned}
$$


Furthermore, $J_{11} \geq J_{111}-\left|J_{112}\right|$, with

$$
\begin{aligned}
J_{111}= & \sum_{k=0}^{\infty} \int_{t-\gamma}^{t} \mathrm{~d} r \sum_{i=1}^{d} v_{i}^{2} \sigma^{2}\left(\Phi^{h}\left(r, x_{i}\right)\right)\left\langle S\left(t-r, x_{i}-*\right), e_{k}(*)\right\rangle_{\mathcal{H}}^{2}, \\
J_{112}= & \sum_{k=0}^{\infty} \int_{t-\gamma}^{t} \mathrm{~d} r \sum_{\substack{i, j=1 \\
i \neq j}}^{d} v_{i} v_{j} \sigma\left(\Phi^{h}\left(r, x_{i}\right)\right) \sigma\left(\Phi^{h}\left(r, x_{j}\right)\right) \\
& \times\left\langle S\left(t-r, x_{i}-*\right), e_{k}(*)\right\rangle_{\mathcal{H}}\left\langle S\left(t-r, x_{j}-*\right), e_{k}(*)\right\rangle_{\mathcal{H}} .
\end{aligned}
$$

The lower bound assumption in (i') and (iv') yield

$$
J_{111} \geq C \gamma^{1+a_{1}}
$$

Parseval's identity and the growth condition on $\sigma$ yield

$$
\left|J_{112}\right| \leq C \sup _{(s, x) \in[0, T] \times \mathbb{R}^{2}}\left(1+\left|\Phi^{h}(s, x)\right|\right)^{2} \int_{t-\gamma}^{t}\left\langle S\left(t-r, x_{i}-*\right), S\left(t-r, x_{j}-*\right)\right\rangle_{\mathcal{H}} \mathrm{d} r
$$

with $i \neq j$. Notice that $\sup _{(s, x) \in[0, T] \times \mathbb{R}^{2}}\left|\Phi^{h}(t, x)\right| \leq C$,

Therefore, as has been checked in the proof of Lemma 5 ,

$$
\left|J_{112}\right| \leq C \gamma^{3}
$$

Then (A.6) and (A.7) yield

$$
\left|J_{11}\right| \geq C\left(\gamma^{1+a_{1}}-\gamma^{3}\right)
$$

Following the same ideas as in the proof of Theorem 2.2 in [8] one can prove that $\sup _{t \in[0, T]}$ $\sup _{|y-z| \leq \xi}\left(\left|\Phi^{h}(t, y)-\Phi^{h}(t, z)\right|\right) \leq C \xi^{\alpha}$, with $\alpha \in\left(0, \frac{1}{2}\left(a_{2} \wedge 1\right)\right)$. Hence

$$
\begin{aligned}
J_{12} & \leq C \gamma^{2 \alpha} \sup \left\{\int_{t-\gamma}^{t}\left\langle S\left(t-r, x_{i}-*\right), S\left(t-r, x_{j}-*\right)\right\rangle_{\mathcal{H}} \mathrm{d} r ; i, j=1, \ldots, d\right\} \\
& \leq C \gamma^{2 \alpha+1+a_{2}} .
\end{aligned}
$$

Consequently (A.8) and (A.9) imply

$$
J_{1} \geq C\left(\gamma^{1+a_{1}}-\gamma^{3}-\gamma^{2 \alpha+1+a_{2}}\right)
$$


Upper bound for $J_{2}$ : By the definition of $\psi_{r, k}(t, x)$ and $J_{2}$, we clearly have $J_{2} \leq C\left(J_{21}+J_{22}\right)$ with

$$
\begin{aligned}
J_{21}= & \sum_{k=0}^{\infty} \int_{t-\gamma}^{t} \mathrm{~d} r \sum_{i=1}^{d} v_{i}^{2}\left(\sum _ { j = 0 } ^ { \infty } \int _ { r } ^ { t } \left\langleS\left(t-s, x_{i}-*\right) \sigma^{\prime}\left(\Phi^{h}(s, *)\right) D_{r, k} \Phi^{h}(s, *),\right.\right. \\
& \left.\left.e_{j}(*)\right\rangle_{\mathcal{H}} h_{j}(s) \mathrm{d} s\right)^{2}, \\
J_{22}= & \sum_{k=0}^{\infty} \int_{t-\gamma}^{t} \mathrm{~d} r \sum_{i=1}^{d} v_{i}^{2}\left(\int_{r}^{t} \mathrm{~d} s \int_{\mathbb{R}^{2}} \mathrm{~d} y S\left(t-s, x_{i}-y\right) b^{\prime}\left(\Phi^{h}(s, y)\right) D_{r, k} \Phi^{h}(s, y)\right)^{2} .
\end{aligned}
$$

Schwarz's inequality and Parseval's identity ensure

$$
J_{21} \leq C \sum_{k=0}^{\infty} \int_{t-\gamma}^{t} \mathrm{~d} r \sum_{i=1}^{d} v_{i}^{2} \int_{r}^{t}\left\|S\left(t-s, x_{i}-*\right) \sigma^{\prime}\left(\Phi^{h}(s, *)\right) D_{r, k} \Phi^{h}(s, *)\right\|_{\mathcal{H}}^{2} \mathrm{~d} s .
$$

Then, applying Fubini's theorem and Schwarz's inequality this term is bounded by

$$
C\left(\sup _{(s, y) \in[t-\gamma, t] \times \mathbb{R}^{2}} \sum_{k=1}^{\infty} \int_{t-\gamma}^{t}\left\|D_{r, k} \Phi^{h}(s, y)\right\|_{\mathcal{H}}^{2} \mathrm{~d} r\right) \mu(\gamma)
$$

Following the proof of Theorem 2.2 in [10] we obtain $J_{21} \leq C \mu(\gamma)^{2}$. Then, by (A.4),

$$
J_{2,1} \leq C \gamma^{2\left(a_{2}+1\right)}
$$

Jensen's inequality, Fubini's theorem and similar arguments as those used to obtain (A.11) yield

$$
J_{22} \leq C \mu(\gamma) \nu(\gamma) \leq C \gamma^{3+a_{2}}
$$

Therefore, by (A.11, A.12)

$$
J_{2} \leq C\left(\gamma^{2\left(a_{2}+1\right)}+\gamma^{3+a_{2}}\right)
$$

Finally (A.10) and (A.13) yield

$$
v^{*} \gamma_{\Phi^{h}(t, \underline{x})} v \geq C\left(\gamma^{1+a_{1}}-\gamma^{3}-\gamma^{2 \alpha+1+a_{2}}-\gamma^{2\left(a_{2}+1\right)}-\gamma^{3+a_{2}}\right)
$$

Set $\gamma=\varepsilon^{\delta}, \delta>0$, such that

$$
\delta\left(1+a_{1}\right)<1,3 \delta>1, \delta\left(2 \alpha+1+a_{2}\right)>1,2 \delta\left(a_{2}+1\right)>1, \delta\left(3+a_{2}\right)>1,
$$

where $\alpha \in\left(0, \frac{1}{2}\left(a_{2} \wedge 1\right)\right)$ and $a_{1}, a_{2}$ satisfy the restrictions stated in (i'). It is easy to check that such a choice is possible. Then, the right hand-side of (A.14) is strictly positive. This finishes the proof of the proposition. $\square$

\section{REFERENCES}

[1] S. Aida, S. Kusuoka and D. Stroock, On the support of Wiener functionals, edited by K.D. Elworthy and N. Ikeda, Asymptotic Problems in Probability Theory: Wiener Functionals and Asymptotics. Longman Scient. and Tech., New York, Pitman Res. Notes in Math. Ser. 284 (1993) 3-34. 
[2] V. Bally and E. Pardoux, Malliavin Calculus for white-noise driven parabolic spde's. Potential Anal. 9 (1998) $27-64$.

[3] G. Ben Arous and R. Léandre, Décroissance exponentielle du noyau de la chaleur sur la diagonale (II). Probab. Theory Related Fields 90 (1991) 377-402.

[4] R. Dalang and N. Frangos, The stochastic wave equation in two spatial dimensions. Ann. Probab. 26 (1998) 187-212.

[5] O. Lévêque, Hyperbolic stochastic partial differential equations driven by boundary noises. Thèse EPFL, Lausanne, 2452 (2001).

[6] D. Mrquez-Carreras, M. Mellouk and M. Sarrà, On stochastic partial differential equations with spatially correlated noise: Smoothness of the law. Stochastic Proc. Appl. 93 (2001) 269-284.

[7] M. Métivier, Semimartingales. De Gruyter, Berlin (1982).

[8] A. Millet and P.-L. Morien, On a stochastic wave equation in two dimensions: Regularity of the solution and its density. Stochastic Proc. Appl. 86 (2000) 141-162.

[9] A. Millet and M. Sanz-Solé, Points of positive density for the solution to a hyperbolic spde. Potential Anal. 7 (1997) $623-659$.

[10] A. Millet and M. Sanz-Solé, A stochastic wave equations in two space dimension: Smoothness of the law. Ann. Probab. 27 (1999) 803-844.

[11] A. Millet and M. Sanz-Solé, Approximation and support theorem for a two space-dimensional wave equation. Bernoulli 6 (2000) 887-915.

[12] P.-L. Morien, Hölder and Besov regularity of the density for the solution of a white-noise driven parabolic spde. Bernoulli 5 (1999) 275-298.

[13] D. Nualart, Malliavin Calculus and Related Fields. Springer-Verlag (1995).

[14] D. Nualart, Analysis on the Wiener space and anticipating calculus, in École d'été de Probabilités de Saint-Flour. SpringerVerlag, Lecture Notes in Math. 1690 (1998) 863-901.

[15] M. Sanz-Solé and M. Sarrà, Path properties of a class of Gaussian processes with applications to spde's, in Stochastic Processes, Physics and Geometry: New interplays, edited by F. Gesztesy et al. American Mathematical Society, CMS Conf. Proc. 28 (2000) 303-316.

[16] J.B. Walsh, An introduction to stochastic partial differential equations, in École d'été de Probabilités de Saint-Flour, edited by P.L. Hennequin. Springer-Verlag, Lecture Notes in Math. 1180 (1986) 266-437. 